\title{
Variations on Descents and Inversions in Permutations
}

\author{
Denis Chebikin \\ 1404 Yorkshire Ln, Shakopee, MN 55379, USA \\ chebikin@gmail.com
}

Submitted: Apr 8, 2008; Accepted: Oct 8, 2008; Published: Oct 20, 2008

Mathematics Subject Classification: 05A05; 05A10; 05A15; 05A19

\begin{abstract}
We study new statistics on permutations that are variations on the descent and the inversion statistics. In particular, we consider the alternating descent set of a permutation $\sigma=\sigma_{1} \sigma_{2} \cdots \sigma_{n}$ defined as the set of indices $i$ such that either $i$ is odd and $\sigma_{i}>\sigma_{i+1}$, or $i$ is even and $\sigma_{i}<\sigma_{i+1}$. We show that this statistic is equidistributed with the odd 3-factor set statistic on permutations $\tilde{\sigma}=\sigma_{1} \sigma_{2} \cdots \sigma_{n+1}$ with $\sigma_{1}=1$, defined to be the set of indices $i$ such that the triple $\sigma_{i} \sigma_{i+1} \sigma_{i+2}$ forms an odd permutation of size 3. We then introduce Mahonian inversion statistics corresponding to the two new variations of descents and show that the joint distributions of the resulting descent-inversion pairs are the same, establishing a connection to two classical Mahonian statistics, MAJ and STAT, along the way. We examine the generating functions involving alternating Eulerian polynomials, defined by analogy with the classical Eulerian polynomials $\sum_{\sigma \in \mathfrak{S}_{n}} t^{\operatorname{des}(\sigma)+1}$ using alternating descents. For the alternating descent set statistic, we define the generating polynomial in two non-commutative variables by analogy with the $a b$-index of the Boolean algebra $B_{n}$, providing a link to permutations without consecutive descents. By looking at the number of alternating inversions, which we define in the paper, in alternating (down-up) permutations, we obtain a new $q$-analog of the Euler number $E_{n}$ and show how it emerges in a $q$-analog of an identity expressing $E_{n}$ as a weighted sum of Dyck paths.
\end{abstract}

\section{Introduction}

Specifying the descent set of a permutation can be thought of as giving information on how the elements are ordered locally, namely, which pairs of consecutive elements are ordered properly and which are not, the latter constituting the descents. The original idea that became the starting point of this research was to generalize descent sets to indicators of relative orders of $k$-tuples of consecutive elements, the next simplest case 
being $k=3$. In this case there are 6 possible relative orders, and thus the analog of the descent set enumerator $\Psi_{n}(\mathbf{a}, \mathbf{b})$, also known as the $a b$-index of the Boolean algebra $B_{n}$, would involve 6 non-commuting variables. In order to defer overcomplication, to keep the number of variables at 2, and to stay close to classical permutation statistics, we can divide triples of consecutive elements into merely "proper" or "improper", defined as having the relative order of an even or an odd permutation of size 3, respectively. We call the improper triples odd 3-factors, and denote the set of positions at which odd 3-factors occur in a permutation $\sigma$ by $D_{3}(\sigma)$. Thus we obtain a concept generalizing classical permutation descents, which could by analogy be called odd 2-factors. It would certainly be very interesting to develop a general theory around the odd $k$-factor statistic, but in this paper we only focus on the $k=3$ case.

Computing the number of permutations with a given odd 3 -factor set $S$ yields a few immediate observations. For example, the number of permutations $\sigma \in \mathfrak{S}_{n}$ with $D_{3}(\sigma)$ equal to a fixed subset $S \subseteq[n-2]$ is divisible by $n$. This fact becomes clear upon the realization that $D_{3}(\sigma)$ is preserved when the elements of $\sigma$ are cyclically shifted, so that 1 becomes 2, 2 becomes 3, and so on. As a result, it makes sense to focus on the set $\tilde{\mathfrak{S}}_{n}$ of permutations of $[n]$ with the first element equal to 1 . A second, less trivial observation arising from early calculations is that the number of permutations in $\tilde{\mathfrak{S}}_{n}$ whose odd 3factor set is empty is the Euler number $E_{n-1}$.

This second observation follows from the equidistribution of the statistic $D_{3}$ on the set $\tilde{\mathfrak{S}}_{n+1}$ with another variation on the descent set statistic, this time on $\mathfrak{S}_{n}$, which we call the alternating descent set (Theorem 2.3). It is defined as the set of positions $i$ at which the permutation has an alternating descent, which is a regular descent if $i$ is odd or an ascent if $i$ is even. Thus the alternating descent set $\hat{D}(\sigma)$ of a permutation $\sigma$ is the set of places where $\sigma$ deviates from the alternating pattern.

Many of the results in this paper that were originally motivated by the odd 3 -factor statistic $d_{3}(\sigma)=\left|D_{3}(\sigma)\right|$ are actually given in terms of the alternating descent statistic $\hat{d}(\sigma)=|\hat{D}(\sigma)|$. We show that the alternating Eulerian polynomials, defined as $\hat{A}_{n}(t):=$ $\sum_{\sigma \in \mathfrak{S}_{n}} t^{\hat{d}(\sigma)+1}$ by analogy with the classical Eulerian polynomials, have the generating function

$$
\sum_{n \geq 1} \hat{A}_{n}(t) \cdot \frac{u^{n}}{n !}=\frac{t(1-h(u(t-1)))}{h(u(t-1))-t}
$$

where $h(x)=\tan x+\sec x$, so that the difference with the classical formula (2) below (specialized at $q=1$ ) is only in that the exponential function is replaced by tangent plus secant (Theorem 4.2).

A similar parallel becomes apparent in our consideration of the analog of the well known identity

$$
\frac{A_{n}(t)}{(1-t)^{n+1}}=\sum_{m \geq 1} m^{n} t^{m}
$$

for $\hat{A}_{n}(t)$. Given a formal power series $f(x)=1+\sum_{n \geq 1} a_{n} x^{n} / n$ !, we define the symmetric 
function

$$
g_{f, n}:=\sum_{\gamma \models n}\left(\begin{array}{l}
n \\
\gamma
\end{array}\right) \cdot a_{\gamma_{1}} a_{\gamma_{2}} \cdots M_{\gamma},
$$

where $\gamma$ runs over all compositions of $n$, and

$$
M_{\gamma}:=\sum_{i_{1}<i_{2}<\cdots} x_{i_{1}}^{\gamma_{1}} x_{i_{2}}^{\gamma_{2}} \cdots
$$

Then (1) can be written as

$$
\frac{A_{n}(t)}{(1-t)^{n+1}}=\sum_{m \geq 1} g_{\exp , n}\left(1^{m}\right) \cdot t^{m},
$$

and we have

$$
\frac{\hat{A}_{n}(t)}{(1-t)^{n+1}}=\sum_{m \geq 1} g_{\tan +\sec , n}\left(1^{m}\right) \cdot t^{m}
$$

where $1^{m}$ denotes setting the variables $x_{1}, x_{2}, \ldots, x_{m}$ to 1 and the remaining variables to 0 (Proposition 5.2).

In Section 7 we discuss the generating function $\hat{\Psi}(\mathbf{a}, \mathbf{b})$ for the number of permutations in $\mathfrak{S}_{n}$ with a given alternating descent set $S \subseteq[n-1]$, denoted $\hat{\beta}_{n}(S)$, which is analogous to the generating polynomial $\Psi_{n}(\mathbf{a}, \mathbf{b})$ for the regular descent set statistic mentioned earlier. The polynomial $\Psi_{n}(\mathbf{a}, \mathbf{b})$ can be expressed as the $c d$-index $\Phi_{n}(\mathbf{c}, \mathbf{d})$ of the Boolean algebra $B_{n}$, where $\mathbf{c}=\mathbf{a}+\mathbf{b}$ and $\mathbf{d}=\mathbf{a} \mathbf{b}+\mathbf{b} \mathbf{a}$. We show that $\hat{\Psi}_{n}$ can also be written in terms of $\mathbf{c}$ and $\mathbf{d}$ as $\hat{\Phi}_{n}(\mathbf{c}, \mathbf{d})=\Phi_{n}\left(\mathbf{c}, \mathbf{c}^{2}-\mathbf{d}\right.$ ) (Proposition 7.2), and that the sum of absolute values of the coefficients of this $(\mathbf{c}, \mathbf{d})$-polynomial, which is the evaluation $\Phi_{n}(1,2)$, is the $n$-th term of a notable combinatorial sequence counting permutations in $\mathfrak{S}_{n}$ with no consecutive descents and no descent at the end (Theorem 7.6). This sequence has properties relevant to this work; in particular, the logarithm of the corresponding exponential generating function is an odd function, which is a crucial property of both $e^{x}$ and $\tan x+\sec x$ that emerges repeatedly in the derivations of the results mentioned above. We discuss the similarities with Euler numbers and alternating permutations in Section 8.

It is natural to wonder if the variations of descents introduced thus far can be accompanied by corresponding variations of inversions. For alternating descents it seems reasonable to consider alternating inversions defined in a similar manner as pairs of indices $i<j$ such that either $i$ is odd and the elements in positions $i$ and $j$ form a regular inversion, or else $i$ is even and these two elements do not form a regular inversion. As for odd 3-factors, we define the accompanying 3-inversion statistic, where a 3-inversion is defined as the number of pairs of indices $(i, j)$ such that $i+1<j$ and the elements in positions $i, i+1$, and $j$, taken in this order, constitute an odd permutation of size 3 . Let $\hat{\imath}(\sigma)$ and $i_{3}(\sigma)$ be the number of alternating inversions and 3-inversions of a permutation $\sigma$, respectively. We find that the joint distribution of the pair $(\hat{d}, \hat{\imath})$ of statistics on the set $\mathfrak{S}_{n}$ is identical to the distribution of the pair $\left(d_{3}, i_{3}\right)$ of statistics on the set $\tilde{\mathfrak{S}}_{n+1}$ (Theorem 3.7). 
It is important to note that odd 3-factors and 3-inversions can each be defined as occurrences of a set of generalized permutation patterns: an odd 3-factor is an occurrence of one of the generalized patterns $\{132,213,321\}$, and a 3 -inversion is an occurrence of one of the generalized patterns $\{13-2,21-3,32-1\}$. Connections with results in permutation pattern theory are briefly discussed at the end of Sections 2 and 3.

Stanley [10] derived a generating function for the joint distribution of the classical descent and inversion statistics on $\mathfrak{S}_{n}$ :

$$
1+\sum_{n \geq 1} \sum_{\sigma \in \mathfrak{S}_{n}} t^{d(\sigma)} q^{\operatorname{inv}(\sigma)} \cdot \frac{u^{n}}{[n]_{q} !}=\frac{1-t}{\operatorname{Exp}_{q}(u(t-1))-t},
$$

where $\operatorname{Exp}_{q}(x)=\sum_{n \geq 0} q^{\left(\begin{array}{l}n \\ 2\end{array}\right)} x^{n} /[n]_{q}$ !, and $d(\sigma)$ and $\operatorname{inv}(\sigma)$ denote the number of descents and inversions of $\sigma$, respectively. (Another good reference on the subject is a recent paper [9] of Shareshian and Wachs.) It would be nice to produce an analog of the generating function (2) for these descent-inversion pairs, but this task appears to be challenging, and it is not even clear what form such a generating function should have, as the $q$-factorials in the denominators of (2) are strongly connected to $q$-binomial coefficients, which have a combinatorial interpretation of the number of inversions in a permutation obtained by concatenating two increasing runs of fixed size. Nevertheless the bivariate polynomial $\hat{A}_{n}(t, q):=\sum_{\sigma \in \mathfrak{S}_{n}} t^{\hat{d}(\sigma)} q^{\hat{\imath}(\sigma)}$ seems to be of interest, and in Section 9 we direct our attention to the $q$-polynomials that result if we set $t=0$. This special case concerns up-down permutations and, more precisely, their distribution according to the number of alternating inversions. For down-up permutations this distribution is essentially the same, the only difference being the order of the coefficients in the $q$-polynomial, and for our purposes it turns out to be more convenient to work with down-up permutations, so we use the distribution of $\hat{\imath}$ on them to define a $q$-analog $\hat{E}_{n}(q)$ of Euler numbers. The formal definition we give is

$$
\hat{E}_{n}(q):=q^{-\left\lfloor n^{2} / 4\right\rfloor} \sum_{\sigma \in \operatorname{Alt}_{n}} q^{\hat{\imath}(\sigma)}
$$

where $\mathrm{Alt}_{n}$ is the set of down-up permutations of $[n]$. The polynomial $\hat{E}_{n}(q)$ is monic with constant term equal to the Catalan number $c_{\lfloor n / 2\rfloor}$ (Proposition 9.2), which hints at the possibility to express $\hat{E}_{n}(q)$ as the sum of $c_{\lfloor n / 2\rfloor}$ "nice" polynomials with constant term 1. We discover such an expression in the form of a $q$-analog of a beautiful identity that represents $E_{n}$ as the sum of weighted Dyck paths of length $2\lfloor n / 2\rfloor$. In this identity we imagine Dyck paths as starting at $(0,0)$ and ending at $(2\lfloor n / 2\rfloor, 0)$. We set the weight of an up-step to be the level at which that step is situated (the steps that touch the "ground" are at level 1, the steps above them are at level 2, and so on) and the weight of a down-step to be either the level of the step (for even $n$ ) or one plus the level of the step (for odd $n$ ). We set the weight of the path to be the product of the weights of all its steps. The sum of the weights taken over all $c_{\lfloor n / 2\rfloor}$ paths then equals $E_{n}$, and if we replace the weight of a step with the $q$-analog of the respective integer, we obtain $\hat{E}_{n}(q)$ (Theorem 9.5). 
The original $q=1$ version of the above identity provides a curious connection between Catalan and Euler numbers. A notable difference between these numbers is in the generating functions: one traditionally considers the ordinary generating function for the former and the exponential one for the latter. An interesting and hopefully solvable problem is the following:

Problem 1.1. Find a generating function interpolating between the classical generating functions for Catalan and Euler numbers using the above q-analog $\hat{E}_{n}(q)$ of Euler numbers. More specifically, is there a nice expression for the power series

$$
H(q, x):=\sum_{n \geq 0} \hat{E}_{n}(q) \cdot \frac{x^{n}}{[n]_{q} !},
$$

so that $H(1, x)=\tan x+\sec x$ and

$$
H(0, x)=\sum_{n \geq 0} c_{\lfloor n / 2\rfloor} x^{n}=\frac{(1+x)\left(1-\sqrt{1-4 x^{2}}\right)}{2 x^{2}} ?
$$

\section{Variations on the descent statistic}

Let $\mathfrak{S}_{n}$ be the set of permutations of $[n]=\{1, \ldots, n\}$, and let $\tilde{\mathfrak{S}}_{n}$ be the set of permutations $\sigma_{1} \sigma_{2} \cdots \sigma_{n}$ of $[n]$ such that $\sigma_{1}=1$. For a permutation $\sigma=\sigma_{1} \cdots \sigma_{n}$, define the descent set $D(\sigma)$ of $\sigma$ by $D(\sigma)=\left\{i \mid \sigma_{i}>\sigma_{j}\right\} \subseteq[n-1]$, and set $d(\sigma)=|D(\sigma)|$.

We say that a permutation $\sigma$ has an odd 3-factor at position $i$ if the permutation $\sigma_{i} \sigma_{i+1} \sigma_{i+2}$, viewed as an element of $\mathfrak{S}_{3}$, is odd, namely, is either 132,213 , or 321 . Let $D_{3}(\sigma)$ be the set of positions at which a permutation $\sigma$ has an odd 3-factor, and set $d_{3}(\sigma)=\left|D_{3}(\sigma)\right|$. An important property of the odd 3-factor statistic is the following.

Lemma 2.1. Let $\omega_{n}^{c}$ be the cyclic permutation $(23 \ldots n 1)$, and let $\sigma \in \mathfrak{S}_{n}$. Then $D_{3}(\sigma)=D_{3}\left(\sigma \omega_{n}^{c}\right)$.

Proof. Multiplying $\sigma$ on the right by $\omega_{n}^{c}$ replaces each $\sigma_{i}<n$ by $\sigma_{i}+1$, and the element of $\sigma$ equal to $n$ by 1 . Thus the elements of the triples $\sigma_{i} \sigma_{i+1} \sigma_{i+2}$ that do not include $n$ maintain their relative order under this operation, and in the triples that include $n$, the relative order of exactly two pairs of elements is altered. Thus the odd 3 -factor set of $\sigma$ is preserved.

Corollary 2.2. For all $i, j, k, \ell \in[n]$ and $B \subseteq[n-2]$, the number of permutations $\sigma \in \mathfrak{S}_{n}$ with $D_{3}(\sigma)=B$ and $\sigma_{i}=j$ is the same as the number of permutations with $D_{3}(\sigma)=B$ and $\sigma_{k}=\ell$.

Proof. The set $\mathfrak{S}_{n}$ splits into orbits of the form $\left\{\sigma, \sigma \omega_{n}^{c}, \sigma\left(\omega_{n}^{c}\right)^{2}, \ldots, \sigma\left(\omega_{n}^{c}\right)^{n-1}\right\}$, and each such subset contains exactly one permutation with a $j$ in the $i$-th position for all $i, j \in$ $[n]$. 
Next, we define another variation on the descent statistic. We say that a permutation $\sigma=\sigma_{1} \cdots \sigma_{n}$ has an alternating descent at position $i$ if either $\sigma_{i}>\sigma_{i+1}$ and $i$ is odd, or else if $\sigma_{i}<\sigma_{i+1}$ and $i$ is even. Let $\hat{D}(\sigma)$ be the set of positions at which $\sigma$ has an alternating descent, and set $\hat{d}(\sigma)=|\hat{D}(\sigma)|$.

Our first result relates the last two statistics by asserting that the odd 3 -factor sets of permutations in $\tilde{\mathfrak{S}}_{n+1}$ are equidistributed with the alternating descent sets of permutations in $\mathfrak{S}_{n}$.

Theorem 2.3. Let $B \subseteq[n-1]$. The number of permutations $\sigma \in \tilde{\mathfrak{S}}_{n+1}$ with $D_{3}(\sigma)=B$ is equal to the number of permutations $\omega \in \mathfrak{S}_{n}$ with $\hat{D}(\omega)=B$.

Proof (by Pavlo Pylyavskyy, private communication). We construct a bijection between $\tilde{\mathfrak{S}}_{n+1}$ and $\mathfrak{S}_{n}$ mapping permutations with odd 3-factor set $B$ to permutations with alternating descent set $B$.

Start with a permutation in $\sigma \in \tilde{\mathfrak{S}}_{n}$. We construct the corresponding permutation $\omega$ in $\mathfrak{S}_{n}$ by the following procedure. Consider $n+1$ points on a circle, and label them with numbers from 1 to $n+1$ in the clockwise direction. For convenience, we refer to these points by their labels. For $1 \leq i \leq n$, draw a line segment connecting $\sigma_{i}$ and $\sigma_{i+1}$. The segment $\sigma_{i} \sigma_{i+1}$ divides the circle into two arcs. Define the sequence $C_{1}, \ldots, C_{n}$, where $C_{i}$ is one of the two arcs between $\sigma_{i}$ and $\sigma_{i+1}$, according to the following rule. Choose $C_{1}$ to be the arc between $\sigma_{1}$ and $\sigma_{2}$ corresponding to going from $\sigma_{1}$ to $\sigma_{2}$ in the clockwise direction. For $i>1$, given the choice of $C_{i-1}$, let $C_{i}$ be the arc between $\sigma_{i}$ and $\sigma_{i+1}$ that either contains or is contained in $C_{i-1}$. The choice of such an arc is always possible and unique. Let $\ell(i)$ denote how many of the $i$ points $\sigma_{1}, \ldots, \sigma_{i}$, including $\sigma_{i}$, are contained in $C_{i}$.

Now, construct the sequence of permutations $\omega^{(i)}=\omega_{1}^{(i)} \ldots \omega_{i}^{(i)} \in \mathfrak{S}_{i}, 1 \leq i \leq n$, as follows. Let $\omega^{(1)}=\ell(1)$. Given $\omega^{(i-1)}$, set $\omega_{i}^{(i)}=\ell(i)$, and let $\omega_{1}^{(i)} \ldots \omega_{i-1}^{(i)}$ be the permutation obtained from $\omega^{(i-1)}$ by adding 1 to all elements which are greater than or equal to $\ell_{i}$. Finally, set $\omega=\omega^{(n)}$.

Next, we argue that the map $\sigma \mapsto \omega$ is a bijection. Indeed, from the subword $\omega_{1} \ldots \omega_{i}$ of $\omega$ one can recover $\ell(i)$ since $\omega_{i}$ is the $\ell(i)$-th smallest element of the set $\left\{\omega_{1}, \ldots, \omega_{i}\right\}$. Then one can reconstruct one by one the arcs $C_{i}$ and the segments connecting $\sigma_{i}$ and $\sigma_{i+1}$ as follows. If $\ell(i)>\ell(i-1)$ then $C_{i}$ contains $C_{i-1}$, and if $\ell(i) \leq \ell(i-1)$ then $C_{i}$ is contained in $C_{i-1}$. Using this observation and the number $\ell(i)$ of the points $\sigma_{1}, \ldots, \sigma_{i}$ contained in $C_{i}$, one can determine the position of the point $\sigma_{i+1}$ relative to the points $\sigma_{1}, \ldots, \sigma_{i}$.

It remains to check that $D_{3}(\sigma)=\hat{D}(\omega)$. Observe that $\sigma$ has a odd 3-factor in position $i$ if and only if the triple of points $\sigma_{i}, \sigma_{i+1}, \sigma_{i+2}$ on the circle is oriented counterclockwise. Also, observe that $\omega_{i}>\omega_{i-1}$ if and only if $C_{i-1} \subset C_{i}$. Finally, note that $C_{i-1} \subset C_{i} \supset C_{i+1}$ or $C_{i-1} \supset C_{i} \subset C_{i+1}$ if and only if triples $\sigma_{i-1}, \sigma_{i}, \sigma_{i+1}$ and $\sigma_{i}, \sigma_{i+1}, \sigma_{i+2}$ have the same orientation. We now show by induction on $i$ that $i \in D_{3}(\sigma)$ if and only if $i \in \hat{D}(\omega)$. From the choice of $C_{1}$ and $C_{2}$, it follows that $C_{1} \subset C_{2}$ if and only if $\sigma_{3}>\sigma_{2}$, and hence $\omega$ has an 
(alternating) descent at position 1 if and only if $\sigma_{1} \sigma_{2} \sigma_{3}=1 \sigma_{2} \sigma_{3}$ is an odd permutation. Suppose the claim holds for $i-1$. By the above observations, we have $\omega_{i-1}<\omega_{i}>\omega_{i+1}$ or $\omega_{i-1}>\omega_{i}<\omega_{i+1}$ if and only if the permutations $\sigma_{i-1} \sigma_{i} \sigma_{i+1}$ and $\sigma_{i} \sigma_{i+1} \sigma_{i+2}$ have the same sign. In other words, $i-1$ and $i$ are either both contained or both not contained in $\hat{D}(\omega)$ if and only if they are either both contained or both not contained in $D_{3}(\sigma)$. It follows that $i \in D_{3}(\sigma)$ if and only if $i \in \hat{D}(\omega)$.

An important special case of Theorem 2.3 is $B=\varnothing$. A permutation $\sigma \in \mathfrak{S}_{n}$ has $\hat{D}(\sigma)=\varnothing$ if and only if it is an alternating (up-down) permutation, i.e. $\sigma_{1}<\sigma_{2}>\sigma_{3}<$ $\cdots$. The number of such permutations of size $n$ is the Euler number $E_{n}$. Thus we get the following corollary:

Corollary 2.4. (a) The number of permutations in $\tilde{\mathfrak{S}}_{n+1}$ with no odd 3-factors is $E_{n}$.

(b) The number of permutations in $\mathfrak{S}_{n+1}$ with no odd 3 -factors is $(n+1) E_{n}$.

Proof. Part (b) follows from Corollary 2.2: for each $j \in[n+1]$, there are $E_{n}$ permutations in $\mathfrak{S}_{n+1}$ beginning with $j$.

Permutations with no odd 3-factors can be equivalently described as simultaneously avoiding generalized patterns 132, 213, and 321 (meaning, in this case, triples of consecutive elements with one of these relative orders). Corollary 2.4(b) appears in the paper [5] of Kitaev and Mansour on simultaneous avoidance of generalized patterns. Thus the above construction yields a bijective proof of their result.

\section{Variations on the inversion statistic}

In this section we introduce analogs of the inversion statistic on permutations corresponding to the odd 3-factor and the alternating descent statistics introduced in Section 2. First, let us recall the standard inversion statistic. For $\sigma \in \mathfrak{S}_{n}$, let $a_{i}$ be the number of indices $j>i$ such that $\sigma_{i}>\sigma_{j}$, and set $\operatorname{code}(\sigma)=\left(a_{1}, \ldots, a_{n-1}\right)$ and $\operatorname{inv}(\sigma)=a_{1}+\cdots+a_{n-1}$.

For a permutation $\sigma \in \mathfrak{S}_{n}$ and $i \in[n-2]$, let $c_{i}^{3}(\sigma)$ be the number of indices $j>i+1$ such that $\sigma_{i} \sigma_{i+1} \sigma_{j}$ is an odd permutation, and set $\operatorname{code}_{3}(\sigma)=\left(c_{1}^{3}(\sigma), c_{2}^{3}(\sigma), \ldots, c_{n-2}^{3}(\sigma)\right)$. Let $C_{k}$ be the set of $k$-tuples $\left(a_{1}, \ldots, a_{k}\right)$ of non-negative integers such that $a_{i} \leq k+1-i$. Clearly, $\operatorname{code}_{3}(\sigma) \in C_{n-2}$.

Lemma 3.1. Let $\omega_{n}^{c}$ be the cyclic permutation $(23 \ldots n 1)$, and let $\sigma \in \mathfrak{S}_{n}$. Then $\operatorname{code}_{3}(\sigma)=\operatorname{code}_{3}\left(\sigma \omega_{n}^{c}\right)$.

Proof. The proof is analogous to that of Lemma 2.1.

Proposition 3.2. The restriction $\operatorname{code}_{3}: \tilde{\mathfrak{S}}_{n} \rightarrow C_{n-2}$ is a bijection. 
Proof. Since $\left|\tilde{\mathfrak{S}}_{n}\right|=\left|C_{n-2}\right|=(n-1)$ !, it suffices to show that the restriction of code 3 to $\tilde{\mathfrak{S}}_{n}$ is surjective. We proceed by induction on $n$. The claim is trivial for $n=3$. Suppose it is true for $n-1$, and let $\left(a_{1}, \ldots, a_{n-2}\right) \in C_{n-2}$. Let $\tau$ be the unique permutation in $\tilde{\mathfrak{S}}_{n-1}$ such that $\operatorname{code}_{3}(\tau)=\left(a_{2}, \ldots, a_{n-2}\right)$. For $1 \leq \ell \leq n$, let $\ell * \tau$ be the permutation in $\mathfrak{S}_{n}$ beginning with $\ell$ such that the relative order of last $n-1$ elements of $\ell * \tau$ is the same as that of the elements of $\tau$. Setting $\ell=n-a_{1}$ we obtain $\operatorname{code}_{3}(\ell * \tau)=\left(a_{1}, \ldots, a_{n-2}\right)$ since $\ell 1 m$ is an odd permutation if and only if $\ell<m$, and there are exactly $a_{1}$ elements of $\ell * \tau$ that are greater than $\ell$. Finally, by Lemma 3.1, the permutation $\sigma=(\ell * \tau)\left(\omega_{n}^{c}\right)^{1-a_{1}} \in \tilde{\mathfrak{S}}_{n}$ satisfies $\operatorname{code}_{3}(\sigma)=\left(a_{1}, \ldots, a_{n-2}\right)$.

Let $i_{3}(\sigma)=c_{1}^{3}(\sigma)+c_{2}^{3}(\sigma)+\cdots+c_{n-2}^{3}(\sigma)$. An immediate consequence of Proposition 3.2 is that $i_{3}(1 * \sigma)$ is a Mahonian statistic on permutations $\sigma \in \mathfrak{S}_{n}$ :

Corollary 3.3. We have

$$
\sum_{\sigma \in \mathfrak{S}_{n}} q^{i_{3}(1 * \sigma)}=(1+q)\left(1+q+q^{2}\right) \cdots\left(1+q+q^{2}+\cdots+q^{n-1}\right)
$$

For a permutation $\sigma \in \mathfrak{S}_{n}$ and $i \in[n-1]$, define $\hat{c}_{i}(\sigma)$ to be the number of indices $j>i$ such that $\sigma_{i}>\sigma_{j}$ if $i$ is odd, or the number of indices $j>i$ such that $\sigma_{i}<\sigma_{j}$ if $i$ is even. Set $\operatorname{code}(\sigma)=\left(\hat{c}_{1}(\sigma), \ldots, \hat{c}_{n-1}(\sigma)\right) \in C_{n-1}$ and $\hat{\imath}(\sigma)=\hat{c}_{1}(\sigma)+\cdots+\hat{c}_{n-1}(\sigma)$.

Proposition 3.4. The map côde : $\mathfrak{S}_{n} \rightarrow C_{n-1}$ is a bijection.

Proof. The proposition follows easily from the fact that if $\operatorname{code}(\sigma)=\left(a_{1}, \ldots, a_{n-1}\right)$ is the standard inversion code of $\sigma$, then $\operatorname{code}(\sigma)=\left(a_{1}, n-2-a_{2}, a_{3}, n-4-a_{4}, \ldots\right)$. Since the standard inversion code is a bijection between $\mathfrak{S}_{n}$ and $C_{n-1}$, so is code.

Corollary 3.5. We have

$$
\sum_{\sigma \in \mathfrak{S}_{n}} q^{\hat{\imath}(\sigma)}=(1+q)\left(1+q+q^{2}\right) \cdots\left(1+q+q^{2}+\cdots+q^{n-1}\right)
$$

Another way to deduce Corollary 3.5 is via the bijection $\sigma \leftrightarrow \sigma^{\vee}$, where

$$
\sigma^{\vee}=\sigma_{1} \sigma_{3} \sigma_{5} \cdots \sigma_{6} \sigma_{4} \sigma_{2}
$$

Proposition 3.6. We have $\hat{\imath}(\sigma)=\operatorname{inv}\left(\sigma^{\vee}\right)$.

Proof. It is easy to verify that a pair $\left(\sigma_{i}, \sigma_{j}\right), i<j$, contributes to $\hat{\imath}(\sigma)$ if and only if it contributes to $\operatorname{inv}\left(\sigma^{\vee}\right)$.

Next, we prove a fundamental relation between the variants of the descent and the inversion statistics introduced thus far. 
Theorem 3.7. We have

$$
\sum_{\sigma \in \tilde{\mathfrak{S}}_{n+1}} t^{d_{3}(\sigma)} q^{i_{3}(\sigma)}=\sum_{\omega \in \mathfrak{S}_{n}} t^{\hat{d}(\omega)} q^{\hat{\imath}(\omega)} .
$$

Proof. The theorem is a direct consequence of the following proposition.

Proposition 3.8. If $\operatorname{code}_{3}(\sigma)=\operatorname{code}(\omega)$ for some $\sigma \in \tilde{\mathfrak{S}}_{n+1}$ and $\omega \in \mathfrak{S}_{n}$, then $D_{3}(\sigma)=$ $\hat{D}(\omega)$.

Proof. The alternating descent set of $\omega$ can be obtained from $\operatorname{code}(\omega)$ as follows:

Lemma 3.9. For $\omega \in \mathfrak{S}_{n}$, write $\left(a_{1}, \ldots, a_{n-1}\right)=\hat{\operatorname{code}}(\omega)$, and set $a_{n}=0$. Then $\hat{D}(\omega)=$ $\left\{i \in[n-1] \mid a_{i}+a_{i+1} \geq n-i\right\}$.

Proof. Suppose $i$ is odd; then if $\omega_{i}>\omega_{i+1}$, i.e. $i \in \hat{D}(\omega)$, then for each $j>i$ we have $\omega_{i}>\omega_{j}$ or $\omega_{i+1}<\omega_{j}$ or both, so $a_{i}+a_{i+1}$ is not smaller than $n-i$, which is the number of elements of $\omega$ to the right of $\omega_{i}$; if on the other hand $\omega_{i}<\omega_{i+1}$, i.e. $i \notin \hat{D}(\omega)$, then for each $j>i$, at most one of the inequalities $\omega_{i}>\omega_{j}$ and $\omega_{i+1}<\omega_{j}$ holds, and neither inequality holds for $j=i+1$, so $a_{i}+a_{i+1} \leq n-i-1$, which is the number of elements of $\omega$ to the right of $\omega_{i+1}$. The case of even $i$ is analogous.

We now show that the odd 3 -factor set of $\sigma$ can be obtained from $\left(a_{1}, \ldots, a_{n-1}\right)$ in the same way.

Lemma 3.10. For $\sigma \in \tilde{\mathfrak{S}}_{n+1}$, write $\left(a_{1}, \ldots, a_{n-1}\right)=\operatorname{code}_{3}(\sigma)$, and set $a_{n}=0$. Then $D_{3}(\sigma)=\left\{i \in[n-1] \mid a_{i}+a_{i+1} \geq n-i\right\}$.

Proof. Let $B=D_{3}(\sigma)$, and let $\sigma^{\prime}=\sigma\left(\omega_{n+1}^{c}\right)^{1-\sigma_{i}} \in \mathfrak{S}_{n+1}$. Then $\sigma_{i}^{\prime}=1$, and by Lemmas 2.1 and 3.1, we have $D_{3}\left(\sigma^{\prime}\right)=D_{3}(\sigma)=B$ and $\operatorname{code}_{3}\left(\sigma^{\prime}\right)=\operatorname{code}_{3}(\sigma)$.

Suppose that $1=\sigma_{i}^{\prime}<\sigma_{i+1}^{\prime}<\sigma_{i+2}^{\prime}$. Then $i \notin B$, and for each $j>i+2$, at most one of the permutations $\sigma_{i}^{\prime} \sigma_{i+1}^{\prime} \sigma_{j}^{\prime}=1 \sigma_{i+1}^{\prime} \sigma_{j}^{\prime}$ and $\sigma_{i+1}^{\prime} \sigma_{i+2}^{\prime} \sigma_{j}^{\prime}$ is odd, because $1 \sigma_{i+1}^{\prime} \sigma_{j}^{\prime}$ is odd if and only if $\sigma_{i+1}^{\prime}>\sigma_{j}^{\prime}$, and $\sigma_{i+1}^{\prime} \sigma_{i+2}^{\prime} \sigma_{j}^{\prime}$ is odd if and only if $\sigma_{i+1}^{\prime}<\sigma_{j}^{\prime}<\sigma_{i+2}^{\prime}$. Hence $a_{i}+a_{i+1}$ is at most $n-1-i$, which is the number of indices $j \in[n+1]$ such that $j>i+2$.

Now suppose that $1=\sigma_{i}^{\prime}<\sigma_{i+1}^{\prime}>\sigma_{i+2}^{\prime}$. Then $i \in B$, and for each $j>i+2$, at least one of the permutations $\sigma_{i}^{\prime} \sigma_{i+1}^{\prime} \sigma_{j}^{\prime}=1 \sigma_{i+1}^{\prime} \sigma_{j}^{\prime}$ and $\sigma_{i+1}^{\prime} \sigma_{i+2}^{\prime} \sigma_{j}^{\prime}$ is odd, because $\sigma_{i+1}^{\prime}>\sigma_{j}^{\prime}$ makes $1 \sigma_{i+1}^{\prime} \sigma_{j}^{\prime}$ odd, and $\sigma_{i+1}^{\prime}<\sigma_{j}$ makes $\sigma_{i+1}^{\prime} \sigma_{i+2}^{\prime} \sigma_{j}^{\prime}$ odd. Thus each index $j>i+1$ contributes to at least one of $a_{i}$ and $a_{i+1}$, so $a_{i}+a_{i+1} \geq n-i$, which is the number of indices $j \in[n+1]$ such that $j>i+1$.

Proposition 3.8 follows from Lemmas 3.9 and 3.10 . 
Combining the results of the above discussion, we conclude that both polynomials of Theorem 3.7 are equal to

$$
\sum_{\left(a_{1}, \ldots, a_{n-1}\right) \in C_{n-1}} t^{\left|\hat{D}\left(a_{1}, \ldots, a_{n-1}\right)\right|} q^{a_{1}+\cdots+a_{n-1}},
$$

where $\hat{D}\left(a_{1}, \ldots, a_{n-1}\right)=\left\{i \in[n-1] \mid a_{i}+a_{i+1} \geq n-i\right\}$.

Note that the bijective correspondence

$$
\sigma \in \mathfrak{S}_{n} \stackrel{\text { code }}{\longrightarrow} c \in C_{n-1} \stackrel{\left(\operatorname{code}_{3}\right)^{-1}}{\longrightarrow} \omega \in \tilde{\mathfrak{S}}_{n+1}
$$

satisfying $\hat{D}(\sigma)=D_{3}(\omega)$ yields another bijective proof of Theorem 2.3.

Besides the inversion statistic, the most famous Mahonian statistic on permutations is the major index. For $\sigma \in \mathfrak{S}_{n}$, define the major index of $\sigma$ by

$$
\operatorname{maj}(\sigma)=\sum_{i \in D(\sigma)} i
$$

Our next result reveals a close relation between the major index and the 3 -inversion statistic $i_{3}$.

Proposition 3.11. For $\sigma \in \mathfrak{S}_{n}$, write $\sigma^{r c}=\sigma_{n}^{\prime} \cdots \sigma_{2}^{\prime} \sigma_{1}^{\prime}$, where $\sigma_{i}^{\prime}=n+1-\sigma_{i}$. Then

$$
i_{3}(1 * \sigma)=\operatorname{maj}\left(\sigma^{r c}\right) \text {. }
$$

Proof. Let $\sigma=1 * \omega \in \tilde{\mathfrak{S}}_{n+1}$. Let $D(\sigma)=\left\{b_{1}<\cdots<b_{d}\right\}$. Write $\sigma=\tau^{(1)} \tau^{(2)} \cdots \tau^{(d+1)}$, where $\tau^{(k)}=\sigma_{b_{k-1}+1} \sigma_{b_{k-1}+2} \cdots \sigma_{b_{k}}$ and $b_{0}=0$ and $b_{d+1}=n$. In other words, we split $\sigma$ into ascending runs between consecutive descents. Fix an element $\sigma_{j}$ of $\sigma$, and suppose $\sigma_{j} \in \tau^{(k)}$. We claim that there are exactly $k-1$ indices $i<j-1$ such that $\sigma_{i} \sigma_{i+1} \sigma_{j}$ is an odd permutation. For each ascending run $\tau^{(\ell)}, \ell<k$, there is at most one element $\sigma_{i} \in \tau^{(\ell)}$ such that $\sigma_{i}<\sigma_{j}<\sigma_{i+1}$, in which case $\sigma_{i} \sigma_{i+1} \sigma_{j}$ is odd. There is no such element in $\tau^{(\ell)}$ if and only if the first element $\sigma_{b_{\ell-1}+1}$ of $\tau^{(\ell)}$ is greater than $\sigma_{j}$, or the last element $\sigma_{b_{\ell}}$ of $\tau^{(\ell)}$ is smaller than $\sigma_{j}$. In the former case we have $\sigma_{b_{\ell}-1}>\sigma_{b_{\ell}}>\sigma_{j}$, so $\sigma_{b_{\ell}-1} \sigma_{b_{\ell}} \sigma_{j}$ is odd, and in the latter case, $\sigma_{j}>\sigma_{b_{\ell}}>\sigma_{b_{\ell}+1}$, so $\sigma_{b_{\ell}} \sigma_{b_{\ell}+1} \sigma_{j}$ is odd. Thus we obtain a one-to-one correspondence between the $k-1$ ascending runs $\tau^{(1)}, \ldots, \tau^{(k-1)}$ and elements $\sigma_{i}$ such that $\sigma_{i} \sigma_{i+1} \sigma_{j}$ is an odd permutation.

We conclude that for each $\tau^{(k)}$, there are $(k-1) \cdot\left(b_{k}-b_{k-1}\right)$ odd triples $\sigma_{i} \sigma_{i+1} \sigma_{j}$ with $\sigma_{j} \in \tau^{(k)}$, and hence

$$
\begin{aligned}
i_{3}(\sigma) & =\sum_{k=1}^{d+1}(k-1) \cdot\left(b_{k}-b_{k-1}\right) \\
& =\left(b_{d+1}-b_{d}\right)+\left(b_{d+1}-b_{d}+b_{d}-b_{d-1}\right)+\left(b_{d+1}-b_{d}+b_{d}-b_{d-1}+b_{d-1}-b_{d-2}\right)+\cdots \\
& =\sum_{m=1}^{d}\left(n-b_{m}\right) .
\end{aligned}
$$


We have $D(\omega)=\left\{b_{1}-1, b_{2}-1, \ldots, b_{d}-1\right\}$, from where it is not hard to see that $D\left(\omega^{r c}\right)=\left\{n-b_{d}, n-b_{d-1}, \ldots, n-b_{1}\right\}$. The proposition follows.

Observe that for a permutation $\pi$ with $\pi^{r c}=\pi_{m}^{\prime} \cdots \pi_{1}^{\prime}$, the triple $\pi_{i} \pi_{i+1} \pi_{i+2}$ is odd if and only if the triple $\pi_{i+2} \pi_{i+1} \pi_{i}$ is even, which in turn is the case if and only if the triple $\pi_{i+2}^{\prime} \pi_{i+1}^{\prime} \pi_{i}^{\prime}$ of consecutive elements of $\pi^{r c}$ is odd. Thus $d_{3}(\pi)=d_{3}\left(\pi^{r c}\right)$, and we obtain the following corollary.

Corollary 3.12. We have

$$
\sum_{\sigma \in \tilde{\mathfrak{S}}_{n+1}} t^{d_{3}(\sigma)} q^{i_{3}(\sigma)}=\sum_{\omega \in \mathfrak{S}_{n}} t^{d_{3}(\omega \circ(n+1))} q^{\operatorname{maj}(\omega)},
$$

where $\omega \circ(n+1)$ is the permutation obtained by appending $(n+1)$ to the right of $\omega$.

Proof. To deduce the identity from Proposition 3.11, write $\sigma=1 * \pi$ and set $\omega=\pi^{r c}$, so that $\omega \circ(n+1)=\sigma^{r c}$.

In the language of permutation patterns, the statistic $i_{3}(\sigma)$ can be defined as the total number of occurrences of generalized patterns 13-2, 21-3, and 32-1 in $\sigma$. (An occurrence of a generalized pattern 13-2 in a permutation $\sigma=\sigma_{1} \sigma_{2} \cdots$ is a pair of indices $(i, j)$ such that $i+1<j$ and $\sigma_{i}, \sigma_{i+1}$, and $\sigma_{j}$ have the same relative order as 1,3 , and 2, that is, $\sigma_{i}<\sigma_{j}<\sigma_{i+1}$, and the other two patterns are defined analogously.) In [1] Babson and Steingrímsson mention the Mahonian statistic $\operatorname{STAT}(\sigma)$, which is defined as $i_{3}(\sigma)$ (treated in terms of the aforementioned patterns) plus $d(\sigma)$. In the permutation $\sigma \circ(n+1)$, where $\sigma \in \mathfrak{S}_{n}$, the descents of $\sigma$ and the last element $n+1$ constitute all occurrences of the pattern 21-3 involving $n+1$, and hence $i_{3}(\sigma \circ(n+1))=\operatorname{STAT}(\sigma)$.

\section{Variations on Eulerian polynomials}

Having introduced two new descent statistics, it is natural to look at the analog of the Eulerian polynomials representing their common distribution on $\mathfrak{S}_{n}$. First, recall the definition of the classical $n$-th Eulerian polynomial:

$$
A_{n}(t):=\sum_{\sigma \in \mathfrak{S}_{n}} t^{d(\sigma)+1}=\sum_{k=1}^{n} A(n, k) \cdot t^{k}
$$

where $A(n, k)$ is the number of permutations in $\mathfrak{S}_{n}$ with $k-1$ descents. There is a well-known formula for the exponential generating function for Eulerian polynomials:

$$
E(t, u)=\sum_{n \geq 1} A_{n}(t) \cdot \frac{u^{n}}{n !}=\frac{t\left(1-e^{u(t-1)}\right)}{e^{u(t-1)}-t} .
$$


In this section we consider analogs of Eulerian numbers and polynomials for our variations of the descent statistic. Define the alternating Eulerian polynomials $\hat{A}_{n}(t)$ by

$$
\hat{A}_{n}(t):=\sum_{\sigma \in \mathfrak{S}_{n}} t^{\hat{d}(\sigma)+1}=\sum_{k=1}^{n} \hat{A}(n, k) \cdot t^{k}
$$

where $\hat{A}(n, k)$ is the number of permutations in $\mathfrak{S}_{n}$ with $k-1$ alternating descents. Our next goal is to find an expression for the exponential generating function

$$
F(t, u):=\sum_{n \geq 1} \hat{A}_{n}(t) \cdot \frac{u^{n}}{n !} .
$$

We begin by deducing a formula for the number of permutations in $\mathfrak{S}_{n}$ with a given alternating descent set. For $S \subseteq[n-1]$, let $\hat{\beta}_{n}(S)$ be the number of permutations $\sigma \in \mathfrak{S}_{n}$ with $\hat{D}(\sigma)=S$, and let $\hat{\alpha}_{n}(S)=\sum_{T \subseteq S} \hat{\beta}_{n}(T)$ be the number of permutations $\sigma \in \mathfrak{S}_{n}$ with $\hat{D}(\sigma) \subseteq S$. For $S=\left\{s_{1}<\cdots<s_{k}\right\} \subseteq[n-1]$, let co $(S)$ be the composition $\left(s_{1}, s_{2}-s_{1}, s_{3}-s_{2}, \ldots, s_{k}-s_{k-1}, n-s_{k}\right)$ of $n$, and for a composition $\gamma=\left(\gamma_{1}, \ldots, \gamma_{\ell}\right)$ of $n$, let $S_{\gamma}$ be the subset $\left\{\gamma_{1}, \gamma_{1}+\gamma_{2}, \ldots, \gamma_{1}+\cdots+\gamma_{\ell-1}\right\}$ of $[n-1]$. Also, define

$$
\left(\begin{array}{l}
n \\
\gamma
\end{array}\right):=\left(\begin{array}{c}
n \\
\gamma_{1}, \ldots, \gamma_{\ell}
\end{array}\right)=\frac{n !}{\gamma_{1} ! \cdots \gamma_{\ell} !}
$$

and

$$
\left(\begin{array}{l}
n \\
\gamma
\end{array}\right)_{E}:=\left(\begin{array}{c}
n \\
\gamma_{1}, \ldots, \gamma_{\ell}
\end{array}\right) \cdot E_{\gamma_{1}} \cdots E_{\gamma_{\ell}}
$$

Lemma 4.1. We have

$$
\hat{\alpha}_{n}(S)=\left(\begin{array}{c}
n \\
\operatorname{co}(S)
\end{array}\right)_{E}
$$

and

$$
\hat{\beta}_{n}(S)=\sum_{T \subseteq S}(-1)^{|S-T|}\left(\begin{array}{c}
n \\
\operatorname{co}(T)
\end{array}\right)_{E} .
$$

Proof. Let $S=\left\{s_{1}<\cdots<s_{k}\right\} \subseteq[n-1]$. Set $s_{0}=0$ and $s_{k+1}=n$ for convenience. The alternating descent set of a permutation $\sigma \in \mathfrak{S}_{n}$ is contained in $S$ if and only if for all $1 \leq i \leq k+1$, the subword $\tau_{i}=\sigma_{s_{i-1}+1} \sigma_{s_{i-1}+2} \cdots \sigma_{s_{i}}$ forms either an up-down (if $s_{i-1}$ is even) or a down-up (if $s_{i-1}$ is odd) permutation. Thus to construct a permutation $\sigma$ with $\hat{D}(\sigma) \subseteq S$, one must choose one of the $\left(\begin{array}{c}n \\ s_{1}-s_{0}, s_{2}-s_{1}, \ldots, s_{k+1}-s_{k}\end{array}\right)=\left(\begin{array}{c}n \\ \operatorname{co}(S)\end{array}\right)$ ways to distribute the elements of $[n]$ among the subwords $\tau_{1}, \ldots, \tau_{k+1}$, and then for each $i \in[k+1]$, choose one of the $E_{s_{i}-s_{i-1}}$ ways of ordering the elements within the subword $\tau_{i}$. The first equation of the lemma follows. The second equation is obtained from the first via the inclusion-exclusion principle. 
Now consider the sum

$$
\sum_{S \subseteq[n-1]}\left(\begin{array}{c}
n \\
\operatorname{co}(S)
\end{array}\right)_{E} x^{|S|}=\sum_{S \subseteq[n-1]} \hat{\alpha}_{n}(S) \cdot x^{|S|}=\sum_{\sigma \in \mathfrak{S}_{n}} \sum_{T \supseteq \hat{D}(\sigma)} x^{|T|}
$$

(a permutation $\sigma$ contributes to $\hat{\alpha}_{n}(T)$ whenever $T \supseteq \hat{D}(\sigma)$ ). The right hand side of (4) is equal to

$$
\begin{aligned}
\sum_{\sigma \in \mathfrak{S}_{n}} \sum_{T \supseteq \hat{D}(\sigma)} x^{\hat{d}(\sigma)+|T-\hat{D}(\sigma)|} & =\sum_{\sigma \in \mathfrak{S}_{n}} x^{\hat{d}(\sigma)} \sum_{i=0}^{n-1-\hat{d}(\sigma)}\left(\begin{array}{c}
n-1-\hat{d}(\sigma) \\
i
\end{array}\right) x^{i} \\
& =\sum_{\sigma \in \mathfrak{S}_{n}} x^{\hat{d}(\sigma)}(1+x)^{n-1-\hat{d}(\sigma)}
\end{aligned}
$$

as there are $\left(\begin{array}{c}n-1-\hat{d}(\sigma) \\ i\end{array}\right)$ subsets of $[n-1]$ containing $\hat{D}(\sigma)$. Continuing with the right hand side of $(5)$, we get

$$
\frac{(1+x)^{n}}{x} \cdot \sum_{\sigma \in \mathfrak{S}_{n}}\left(\frac{x}{1+x}\right)^{\hat{d}(\sigma)+1}=\frac{(1+x)^{n}}{x} \cdot \hat{A}_{n}\left(\frac{x}{1+x}\right) .
$$

Combining equations (4)-(6), we obtain

$$
\sum_{n \geq 1}\left(\sum_{S \subseteq[n-1]}\left(\begin{array}{c}
n \\
\operatorname{co}(S)
\end{array}\right)_{E} x^{|S|}\right) \cdot \frac{y^{n}}{n !}=\frac{1}{x} \cdot \sum_{n \geq 1} \hat{A}_{n}\left(\frac{x}{1+x}\right) \cdot \frac{y^{n}(1+x)^{n}}{n !} .
$$

Since $S \mapsto \operatorname{co}(S)$ is a bijection between $[n-1]$ and the set of compositions of $n$, the left hand side of $(7)$ is

$$
\sum_{n \geq 1}\left(\sum_{\gamma} \frac{E_{\gamma_{1}} \cdots E_{\gamma_{\ell}}}{\gamma_{1} ! \cdots \gamma_{\ell} !} \cdot x^{\ell-1}\right) \cdot y^{n}=\frac{1}{x} \cdot \sum_{\ell \geq 1} x^{\ell} \cdot\left(\sum_{i \geq 1} \frac{E_{i} y^{i}}{i !}\right)^{\ell},
$$

where the inside summation in the left hand side is over all compositions $\gamma=\left(\gamma_{1}, \ldots, \gamma_{\ell}\right)$ of $n$. Applying the well-known formula $\sum_{j \geq 0} E_{j} y^{j} / j !=\tan y+\sec y$, the right hand side of (8) becomes

$$
\frac{1}{x} \cdot \sum_{\ell \geq 1} x^{\ell}(\tan y+\sec y-1)^{\ell}=\frac{1}{x} \cdot\left(\frac{1}{1-x(\tan y+\sec y-1)}-1\right) .
$$

Now set $t=\frac{x}{1+x}$ and $u=y(1+x)$. Equating the right hand sides of (7) and (9), we obtain

$$
F(t, u)=\sum_{n \geq 1} \hat{A}_{n}(t) \cdot \frac{u^{n}}{n !}=\left(\frac{1}{1-x(\tan y+\sec y-1)}-1\right) .
$$


Finally, applying the inverse substitution $x=\frac{t}{1-t}$ and $y=u(1-t)$ and simplifying yields an expression for $F(t, u)$ :

$$
\begin{aligned}
F(t, u) & =\frac{x(\tan y+\sec y-1)}{1-x(\tan y+\sec y-1)} \\
& =\frac{t}{1-t} \cdot\left(\frac{\tan y+\sec y-1}{1-\frac{t}{1-t} \cdot(\tan y+\sec y-1)}\right) \\
& =\frac{t \cdot(\tan (u(1-t))+\sec (u(1-t))-1)}{1-t \cdot(\tan (u(1-t))+\sec (u(1-t)))} .
\end{aligned}
$$

Using the property $(\tan z+\sec z)(\tan (-z)+\sec (-z))=1$, we can rewrite the above expression for $F(t, u)$ as follows:

Theorem 4.2. We have

$$
F(t, u)=\frac{t \cdot(1-\tan (u(t-1))-\sec (u(t-1)))}{\tan (u(t-1))+\sec (u(t-1))-t} .
$$

Thus $F(t, u)$ can be expressed by replacing the exponential function in the formula (3) for $E(t, u)$ by tangent plus secant. In fact, omitting the Euler numbers and working with standard multinomial coefficients gives a proof of (3).

A basic result on Eulerian polynomials is the identity

$$
\frac{A_{n}(t)}{(1-t)^{n+1}}=\sum_{m \geq 1} m^{n} t^{m}
$$

Our next result is a similar identity involving alternating Eulerian polynomials. For a partition $\lambda$ of $n$ with $r_{i}$ parts equal to $i$, define

$$
z_{\lambda}:=1^{r_{1}} \cdot r_{1} ! \cdot 2^{r_{2}} \cdot r_{2} ! \cdots
$$

Theorem 4.3. Let

$$
\hat{f}_{n}(m)=\sum_{\lambda} \frac{n !}{z_{\lambda}} \cdot \frac{E_{\lambda_{1}-1} E_{\lambda_{2}-1} \cdots}{\left(\lambda_{1}-1\right) !\left(\lambda_{2}-1\right) ! \cdots} \cdot m^{\ell(\lambda)},
$$

where the sum is over all partitions $\lambda=\left(\lambda_{1}, \lambda_{2}, \ldots, \lambda_{\ell(\lambda)}\right)$ of $n$ into odd parts. Then

$$
\frac{\hat{A}_{n}(t)}{(1-t)^{n+1}}=\sum_{m \geq 1} \hat{f}_{n}(m) t^{m}
$$

Proof. Let us consider the generating function

$$
G(t, u):=\sum_{n \geq 1} \frac{\hat{A}(t)}{(1-t)^{n+1}} \cdot \frac{u^{n}}{n !} .
$$


Then, by (11), we have

$$
G(t, u)=\frac{1}{1-t} \cdot F\left(t, \frac{u}{1-t}\right)=\frac{t \cdot(\tan u+\sec u-1)}{(1-t)(1-t \cdot(\tan u+\sec u))} .
$$

Define

$$
H(m, u):=\sum_{n \geq 1} \hat{f}_{n}(m) \cdot \frac{u^{n}}{n !} .
$$

This series can be rewritten as follows:

$$
H(m, u)=\sum_{n \geq 1} \frac{\hat{f}_{n}(m)}{n !} \cdot u^{n}=-1+\prod_{i \geq 0} \sum_{j \geq 0} \frac{1}{j !}\left(\frac{E_{2 i} m u^{2 i+1}}{(2 i+1) !}\right)^{j} .
$$

Indeed, for each $i$, the index $j$ in the summation is the number of parts equal to $2 i+1$ in a partition of $n$ into odd parts, and it is not hard to check that the contribution of $j$ parts equal to $2 i+1$ to the appropriate terms of $\hat{f}_{n}(m) / n$ ! is given by the expression inside the summation on the right. We subtract 1 to cancel out the empty partition of 0 counted by the product on the right but not by $H(m, u)$. Continuing with the right hand side of (14), we get

$$
\begin{aligned}
H(m, u)+1 & =\prod_{i \geq 0} \exp \left(\frac{E_{2 i} m u^{2 i+1}}{(2 i+1) !}\right) \\
& =\exp \left(m \sum_{i \geq 0}\left(\frac{E_{2 i} u^{2 i+1}}{(2 i+1) !}\right)\right) .
\end{aligned}
$$

The sum in the right hand side of (15) is the antiderivative of $\sum_{i \geq 0} E_{2 i} u^{2 i} /(2 i) !=\sec u$ that vanishes at $u=0$; this antiderivative is $\ln (\tan u+\sec u)$. Therefore

$$
H(m, u)+1=(\tan u+\sec u)^{m} .
$$

Hence we have

$$
\sum_{m \geq 1} H(m, u) \cdot t^{m}=\frac{(\tan u+\sec u) \cdot t}{1-(\tan u+\sec u) \cdot t}-\frac{1}{1-t} .
$$

It is straightforward to verify that the right hand sides of (13) and (16) agree, and thus

$$
\sum_{n \geq 1} \frac{\hat{A}_{n}(t)}{(1-t)^{n+1}} \cdot \frac{u^{n}}{n !}=G(t, u)=\sum_{m \geq 1} H(m, u) t^{m}=\sum_{m, n \geq 1} \hat{f}_{n}(m) t^{m} \cdot \frac{u^{n}}{n !} .
$$

Equating the coefficients of $u^{n} / n$ ! on both sides of (17) completes the proof of the theorem.

In the terminology of [12, Sec. 4.5], Theorem 4.3 states that the polynomials $\hat{A}_{n}(t)$ are the $\hat{f}_{n}$-Eulerian polynomials. 


\section{$5 \quad$ Eulerian polynomials and symmetric functions}

The results of the previous section can be tied to the theory of symmetric functions. Let us recall some basics. For a composition $\gamma=\left(\gamma_{1}, \gamma_{2}, \ldots, \gamma_{k}\right)$, the monomial quasisymmetric function $M_{\gamma}\left(x_{1}, x_{2}, \ldots\right)$ is defined by

$$
M_{\gamma}:=\sum_{1 \leq i_{1}<\cdots<i_{k}} x_{i_{1}}^{\gamma_{1}} x_{i_{2}}^{\gamma_{2}} \cdots x_{i_{k}}^{\gamma_{k}}
$$

Let $\pi(\gamma)$ denote the partition obtained by rearranging the parts of $\gamma$ in non-increasing order. Then for a partition $\lambda$, the monomial symmetric function $m_{\lambda}\left(x_{1}, x_{2}, \ldots\right)$ is defined as

$$
m_{\lambda}:=\sum_{\gamma: \pi(\gamma)=\lambda} M_{\gamma}
$$

Let $f(x)$ be a function given by the formal power series

$$
f(x)=1+\sum_{n \geq 1} \frac{a_{n} x^{n}}{n !} .
$$

Define the symmetric function $g_{f, n}\left(x_{1}, x_{2}, \ldots\right)$ by

$$
g_{f, n}:=\sum_{\gamma \models n}\left(\begin{array}{l}
n \\
\gamma
\end{array}\right) \cdot a_{\gamma_{1}} a_{\gamma_{2}} \cdots M_{\gamma}=\sum_{\lambda \vdash n}\left(\begin{array}{l}
n \\
\lambda
\end{array}\right) \cdot a_{\lambda_{1}} a_{\lambda_{2}} \cdots m_{\lambda},
$$

where by $\gamma \models n$ and $\lambda \vdash n$ we mean that $\gamma$ and $\lambda$ are a composition and a partition of $n$, respectively. This function can be thought of as the generating function for numbers like $\alpha_{n}(S)$ or $\hat{\alpha}_{n}(S)$ (the number of permutations $\sigma \in \mathfrak{S}_{n}$ with $D(\sigma) \subseteq S$ or $\hat{D}(\sigma) \subseteq S$, respectively). Our first step is to express $g_{f, n}$ in terms of the power sum symmetric functions $p_{k}\left(x_{1}, x_{2}, \ldots\right)=\sum x_{i}^{k}$.

Consider the generating function

$$
G_{f}\left(x_{1}, x_{2}, \ldots ; u\right):=\sum_{n \geq 0} g_{f, n} \cdot \frac{u^{n}}{n !} .
$$

Then we have

$$
G_{f}=\sum_{n \geq 0} \sum_{\gamma \models n} \frac{a_{\gamma_{1}} a_{\gamma_{2}} \cdots}{\gamma_{1} ! \gamma_{2} ! \cdots} \cdot M_{\gamma} u^{n}=\prod_{i \geq 1} f\left(x_{i} u\right)
$$

Now let us write

$$
\ln (f(x))=\sum_{n \geq 1} \frac{b_{n} x^{n}}{n !}
$$

Then from (19) we have

$$
\ln G_{f}=\sum_{i \geq 1} \ln \left(f\left(x_{i} u\right)\right)=\sum_{n \geq 1} b_{n} p_{n}\left(x_{1}, x_{2}, \ldots\right) \cdot \frac{u^{n}}{n !} .
$$


Since the power sum symmetric functions $p_{\lambda}=p_{\lambda_{1}} p_{\lambda_{2}} \cdots$, with $\lambda$ ranging over all partitions of positive integers, form a basis for the ring of symmetric functions, the transformation $p_{n} \mapsto b_{n} p_{n} u^{n} /(n-1)$ !, where $u$ is regarded as a scalar, extends to a homomorphism of this ring. Applying this homomorphism to the well-known identity

$$
\exp \sum_{n \geq 1} \frac{1}{n} \cdot p_{n}=\sum_{\lambda} z_{\lambda}^{-1} p_{\lambda}
$$

where $\lambda$ ranges over all partitions of positive integers, we obtain from (21) that

$$
\begin{aligned}
G_{f} & =\exp \sum_{n \geq 1} \frac{1}{n} \cdot\left(\frac{b_{n} p_{n} u^{n}}{(n-1) !}\right) \\
& =\sum_{\lambda} z_{\lambda}^{-1} \cdot \frac{b_{\lambda_{1}} b_{\lambda_{2}} \cdots}{\left(\lambda_{1}-1\right) !\left(\lambda_{2}-1\right) ! \cdots} \cdot p_{\lambda} u^{|\lambda|} .
\end{aligned}
$$

Comparing the coefficients of $u^{n}$ in (18) and (22), we conclude the following:

Proposition 5.1. For a function $f(x)$ with $f(0)=1$ and $\ln (f(x))=\sum_{n \geq 1} b_{n} x^{n} / n$ ! we have

$$
g_{f, n}=\sum_{\lambda \vdash n} \frac{n !}{z_{\lambda}} \cdot \frac{b_{\lambda_{1}} b_{\lambda_{2}} \cdots}{\left(\lambda_{1}-1\right) !\left(\lambda_{2}-1\right) ! \cdots} \cdot p_{\lambda} .
$$

Two special cases related to earlier discussion are $f(x)=e^{x}$ and $f(x)=\tan x+\sec x$. For $f(x)=e^{x}$, we have $b_{1}=1, b_{2}=b_{3}=\cdots=0$, and hence $g_{f, n}=p_{1}^{n}$. In the case of $f(x)=\tan x+\sec x$, we have

$$
b_{i}= \begin{cases}E_{i-1} & \text { if } i \text { is odd } \\ 0 & \text { if } i \text { is even }\end{cases}
$$

thus the coefficient at $p_{\lambda}$ in the expression of Proposition 5.1 coincides with the coefficient in the term for $\lambda$ in the definition of the polynomial $\hat{f}_{n}(m)$ of Theorem 4.3. These observations lead to the following restatements of the classical identity (12) and Theorem 4.3.

Proposition 5.2. Let $g\left(1^{m}\right)$ denote the evaluation of $g\left(x_{1}, x_{2}, \ldots\right)$ at $x_{1}=x_{2}=\cdots=$ $x_{m}=1, x_{m+1}=x_{m+2}=\cdots=0$. Then

$$
\frac{A_{n}(t)}{(1-t)^{n+1}}=\sum_{m \geq 1} g_{\mathrm{exp}, n}\left(1^{m}\right) \cdot t^{m}
$$

and

$$
\frac{\hat{A}_{n}(t)}{(1-t)^{n+1}}=\sum_{m \geq 1} g_{\tan +\sec , n}\left(1^{m}\right) \cdot t^{m}
$$

Proof. We have $p_{i}\left(1^{m}\right)=m$, and hence $p_{\lambda}\left(1^{m}\right)=m^{\ell(\lambda)}$. 
We conclude the section with a pair of problems.

Problem 5.3. Prove Proposition 5.2 without referring to the results of Section 4.

Observe that for $\gamma=\left(\gamma_{1}, \gamma_{2}, \ldots, \gamma_{k}\right) \models n$, we have $M_{\gamma}\left(1^{m}\right)=\left(\begin{array}{c}m \\ k\end{array}\right)$, the number of monomials $x_{i_{1}}^{\gamma_{1}} x_{i_{2}}^{\gamma_{2}} \cdots x_{i_{k}}^{\gamma_{k}}$ where $1 \leq i_{1}<\cdots<i_{k} \leq m$, which are the monomials in the definition of $M_{\gamma}$ that evaluate to 1 .

It would also be of interest to relate the observations of this section to Schur functions. One possibility is to consider the following generalization of the complete homogeneous symmetric function. Let $\varphi_{f}$ be the homomorphism of the ring of symmetric functions defined by $p_{n} \mapsto b_{n} p_{n} /(n-1)$ !, where the $b_{i}$ 's are as in equation (20). Let

$$
h_{f, n}:=\sum_{\lambda \vdash n} z_{\lambda}^{-1} \varphi_{f}\left(p_{\lambda}\right) .
$$

For $f(x)=(1-x)^{-1}$, the homomorphism $\varphi$ is identity, and $h_{f, n}$ is the standard complete homogeneous symmetric function $h_{n}$, defined to be the sum of all monomials in $x_{1}, x_{2}$, $\ldots$, of degree $n$. Then (22) becomes

$$
G_{f}=\sum_{n \geq 1} h_{f, n} u^{n}
$$

(we do not really need $u$ here because of homegeneity). We can define the generalized Schur function $s_{f, \lambda}$, where $\lambda=\left(\lambda_{1}, \lambda_{2}, \ldots\right) \vdash n$, by the Jacobi-Trudi identity

$$
s_{f, \lambda}:=\operatorname{det}\left[h_{f, \lambda_{i}-i+j}\right]_{1 \leq i, j \leq n},
$$

where $h_{f, 0}=1$ and $h_{f, k}=0$ for $k<0$ (see [13, Sec. 7.16]).

Problem 5.4. What can be said about $s_{f, \lambda}$ for $f(x)=e^{x}$ and $f(x)=\tan x+\sec x$ ?

\section{The alternating Eulerian numbers}

In this section we give a recurrence relation that allows to construct a triangle of alternating Eulerian numbers $\hat{A}(n, k)$ introduced in Section 4. (Recall that $\hat{A}(n, k)$ denotes the number of permutations in $\mathfrak{S}_{n}$ with $k-1$ alternating descents.) The first few rows of this triangle are given in Table 2.1.

The following lemma provides a way to compute alternating Eulerian numbers given the initial condition $\hat{A}(n, 1)=E_{n}$.

Lemma 6.1. For $n \geq k \geq 0$ we have

$$
\begin{aligned}
& \sum_{i=0}^{n} \sum_{j=0}^{k}\left(\begin{array}{l}
n \\
i
\end{array}\right) \cdot \hat{A}(i, j+1) \cdot \hat{A}(n-i, k-j+1) \\
& =(n+1-k) \hat{A}(n, k+1)+(k+1) \hat{A}(n, k+2) .
\end{aligned}
$$




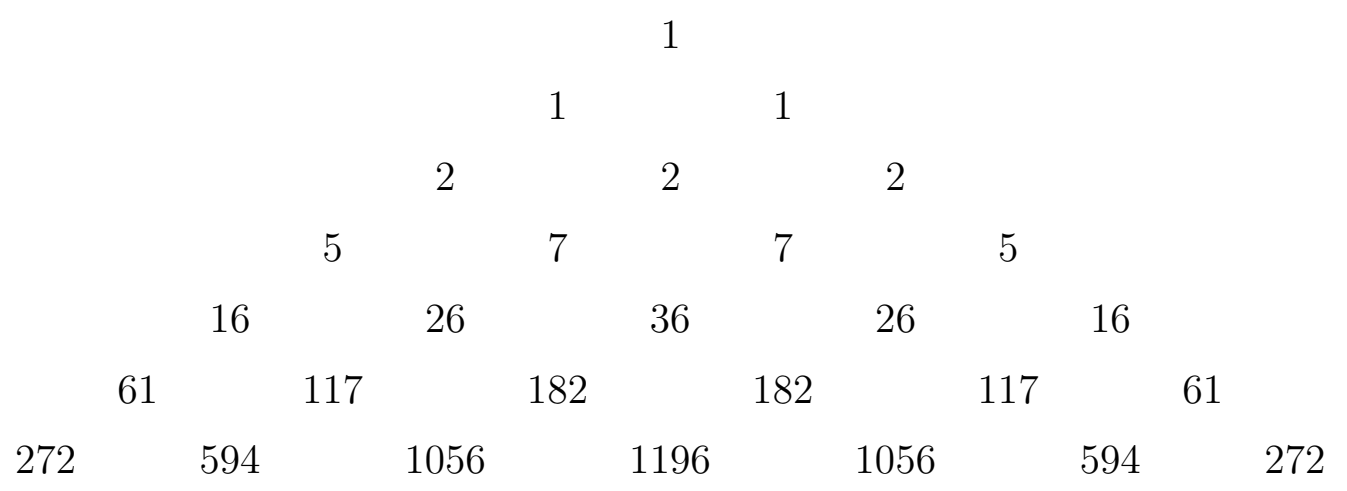

Table 1: Triangle of alternating Eulerian numbers

Proof. First, suppose that $k$ is even. The left hand side of the equation counts the number of ways to split the elements of $[n]$ into two groups of sizes $i$ and $n-i$, arrange the elements in the first and the second group so that the resulting permutations have $j$ and $k-j$ alternating descents, respectively, and writing down the second permutation after the first to form a permtutation of $[n]$. This permutation has either $k$ or $k+1$ alternating descents, depending on whether an alternating descent is produced at position $i$. For a permutation $\sigma \in \mathfrak{S}_{n}$ with $\hat{\imath}(\sigma)=k$, there are exactly $n+1-k$ ways to produce $\sigma$ by means of the above procedure, one for every choice of $i \in \hat{D}(\sigma) \cup\{0, n\}$. Similarly, for $\sigma \in \mathfrak{S}_{n}$ such that $\hat{\imath}(\sigma)=k+1$, there are exactly $k+1$ ways to produce $\sigma$, one for every choice of $i \in \hat{D}(\sigma)$. The identity follows.

As for odd $k$, the same argument is valid, except that the quantity $\hat{A}(n-i, k-j+1)$ in the left hand side should be interpreted as the number of ways to arrange the elements of the second group to form a permutation with $k-j$ alternating ascents, which become alternating descents when the two permutations are concatenated.

Recall the generating function

$$
F(t, u)=\sum_{n, k \geq 1} \hat{A}(n, k) \cdot \frac{t^{k} u^{n}}{n !}
$$

introduced in Section 4. An alternative way to express $F(t, u)$ and obtain the result of Theorem 4.2 is by solving a partial differential equation arising from the recurrence of Lemma 6.1.

Proposition 6.2. The function $F(t, u)$ is the solution of the partial differential equation

$$
F^{2}-F=u \cdot \frac{\partial F}{\partial u}+(1-t) \cdot \frac{\partial F}{\partial t}
$$

with the initial condition $F(0, u)=\tan u+\sec u$. 
Proof. Since $\hat{A}(n, 0)=0$ for all $n$, the left hand side of (23) is $n$ ! times the coefficient of $t^{k} u^{n}$ in $(F(t, u))^{2}$, which we denote by $\left[t^{k} u^{n}\right] F^{2}$. The right hand side of $(23)$ is

$$
\begin{aligned}
& n ! \cdot\left(\frac{\hat{A}(n, k+1)}{(n-1) !}+\frac{\hat{A}(n, k+1)}{n !}-\frac{k \hat{A}(n, k+1)}{n !}+\frac{(k+1) \hat{A}(n, k+2)}{n !}\right) \\
& =n ! \cdot\left(\left[t^{k} u^{n}\right] F_{u}+\left[t^{k} u^{n}\right] F-\left[t^{k-1} u^{n}\right] F_{t}+\left[t^{k} u^{n}\right] F_{t}\right) \\
& =n ! \cdot\left[t^{k} u^{n}\right]\left(u F_{u}+F-t F_{t}+F_{t}\right),
\end{aligned}
$$

where $F_{t}$ and $F_{u}$ denote partial derivatives of $F$ with respect to $t$ and $u$. Equating the above with $n ! \cdot\left[t^{k} u^{n}\right] F^{2}$ proves $(24)$.

\section{The generating function for the alternating descent set statistic}

Besides the generating polynomials for the alternating descent statistic, another natural generating function to consider is one counting permutations by their alternating descent set. We begin by stating some well-known facts about the analogous generating function for the classical descent set statistic.

Fix a positive integer $n$. For a subset $S \subseteq[n-1]$, define the monomial $u_{S}$ in two non-commuting variables $\mathbf{a}$ and $\mathbf{b}$ by $u_{S}=u_{1} u_{2} \cdots u_{n-1}$, where

$$
u_{i}= \begin{cases}\mathbf{a} & \text { if } i \notin S \\ \mathbf{b} & \text { if } i \in S\end{cases}
$$

Consider the generating function

$$
\Psi_{n}(\mathbf{a}, \mathbf{b}):=\sum_{S \subseteq[n-1]} \beta_{n}(S) u_{S}
$$

where $\beta_{n}(S)$ is the number of permutations in $\mathfrak{S}_{n}$ with descent set $S$. The polynomial $\Psi_{n}(\mathbf{a}, \mathbf{b})$ is known as the ab-index of the Boolean algebra $B_{n}$. A remarkable property of $\Psi_{n}(\mathbf{a}, \mathbf{b})$ (and also of $a b$-indices of a wide class of posets, including face lattices of polytopes) is that it can be expressed in terms of the variables $\mathbf{c}=\mathbf{a}+\mathbf{b}$ and $\mathbf{d}=\mathbf{a} \mathbf{b}+\mathbf{b} \mathbf{a}$. The polynomial $\Phi_{n}(\mathbf{c}, \mathbf{d})$ defined by $\Psi_{n}(\mathbf{a}, \mathbf{b})=\Phi_{n}(\mathbf{a}+\mathbf{b}, \mathbf{a} \mathbf{b}+\mathbf{b} \mathbf{a})$ is called the $c d$-index of $B_{n}$.

The polynomial $\Phi_{n}(\mathbf{c}, \mathbf{d})$ has positive integer coefficients, for which several combinatorial interpretations have been found. Here we give one that will help establish a connection with the alternating descent set statistic. We proceed with a definition.

Definition 7.1. A permutation is simsun if, for all $k \geq 0$, removing $k$ largest elements from it results in a permutation with no consecutive descents. 
Let $\mathrm{SS}_{n}$ be the set of simsun permutations in $\mathfrak{S}_{n}$ whose last element is $n$. (Thus $\mathrm{SS}_{n}$ is essentially the set of simsun permutations of $[n-1]$ with an $n$ attached at the end.) It is known that $\left|\mathrm{SS}_{n}\right|=E_{n}$.

For a permutation $\sigma \in \mathrm{SS}_{n}$, define the $(\mathbf{c}, \mathbf{d})$-monomial $\operatorname{cd}(\sigma)$ as follows: write out the descent set of $\sigma$ as a string of pluses and minuses denoting ascents and descents, respectively, and then replace each occurrence of " - + " by $\mathbf{d}$, and each remaining plus by c. This definition is valid because a simsun permutation has no consecutive descents. For example, consider the permutation $423516 \in \mathrm{SS}_{6}$. Its descent set in the above notation is " -++-+ ", and thus $\operatorname{cd}(423516)=\mathbf{d} \mathbf{c d}$.

The simsun permutations provide a combinatorial expression for the $c d$-index of $B_{n}$ :

$$
\Phi_{n}(\mathbf{c}, \mathbf{d})=\sum_{\sigma \in \mathrm{SS}_{n}} \operatorname{cd}(\sigma)
$$

(see for example [11, Prop. 2.2]).

Now let us define the analog of $\Psi_{n}(\mathbf{a}, \mathbf{b})$ for the alternating descent set statistic:

$$
\hat{\Psi}_{n}(\mathbf{a}, \mathbf{b}):=\sum_{S \subseteq[n-1]} \hat{\beta}_{n}(S) u_{S} .
$$

Proposition 7.2. There exists a polynomial $\hat{\Phi}_{n}(\mathbf{c}, \mathbf{d})$ such that

$$
\hat{\Phi}_{n}(\mathbf{a}+\mathbf{b}, \mathbf{a} \mathbf{b}+\mathbf{b} \mathbf{a})=\hat{\Psi}_{n}(\mathbf{a}, \mathbf{b}),
$$

namely, $\hat{\Phi}_{n}(\mathbf{c}, \mathbf{d})=\Phi_{n}\left(\mathbf{c}, \mathbf{c}^{2}-\mathbf{d}\right)$.

Proof. Note that $\hat{\Psi}_{n}(\mathbf{a}, \mathbf{b})$ is the polynomial obtained from $\Psi(\mathbf{a}, \mathbf{b})$ by switching the letters at even positions in all the $(\mathbf{a}, \mathbf{b})$-monomials. For example, we have $\Psi_{3}(\mathbf{a}, \mathbf{b})=$ $\mathbf{a} \mathbf{a}+2 \mathbf{a} \mathbf{b}+2 \mathbf{b} \mathbf{a}+\mathbf{b} \mathbf{b}$, so $\hat{\Psi}_{3}(\mathbf{a}, \mathbf{b})=\mathbf{a} \mathbf{b}+2 \mathbf{a} \mathbf{a}+2 \mathbf{b} \mathbf{b}+\mathbf{b} \mathbf{a}$. In terms of the variables $\mathbf{c}$ and $\mathbf{d}$, this operation corresponds to replacing $\mathbf{d}=\mathbf{a} \mathbf{b}+\mathbf{b} \mathbf{a}$ with $\mathbf{a} \mathbf{a}+\mathbf{b} \mathbf{b}=\mathbf{c}^{2}-\mathbf{d}$, and $\mathbf{c}=\mathbf{a}+\mathbf{b}$ with either $\mathbf{a}+\mathbf{b}$ or $\mathbf{b}+\mathbf{a}$, which in any case is still equal to $\mathbf{c}$.

The polynomial $\hat{\Phi}_{n}(\mathbf{c}, \mathbf{d})$ has both positive and negative coefficients, but the polynomial $\hat{\Phi}_{n}(\mathbf{c},-\mathbf{d})=\Phi_{n}\left(\mathbf{c}, \mathbf{c}^{2}+\mathbf{d}\right)$ has only positive coefficients. It would be nice to give a combinatorial interpretation for these coefficients similar to that of the coefficients of $\Phi_{n}(\mathbf{c}, \mathbf{d})$, so that the coefficients of $\hat{\Phi}_{n}(\mathbf{c},-\mathbf{d})$ enumerate permutations of a certain kind according to some statistic. In what follows we show that the sum of the coefficients of $\hat{\Phi}_{n}(\mathbf{c},-\mathbf{d})$ is equal to the number of permutations containing no consecutive descents and not ending with a descent. Let $\mathcal{R}_{n}$ denote the set of such permutations of $[n]$.

In working with the different kinds of permutations that have emerged thus far we use the approach of min-tree representation of permutations introduced by Hetyei and Reiner [4]. To a word $w$ whose letters are distinct elements of $[n]$, associate a labeled rooted planar binary tree according to the following recursive rule. Let $m$ be the smallest letter 


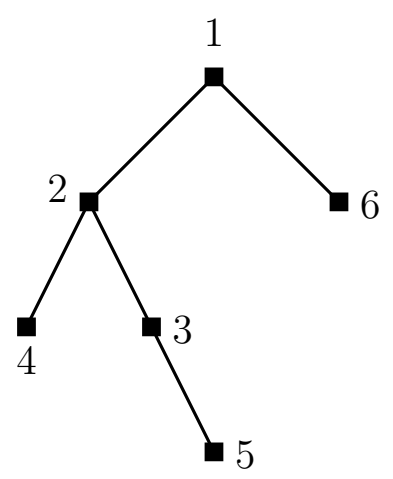

Figure 1: The tree $T(423516)$

of $w$, and write $w=w_{1} \circ m \circ w_{2}$, where $\circ$ denotes concatenation. Then form the tree $T(w)$ by labeling the root with $m$ and setting the left and the right subtrees of the root to be $T\left(w_{1}\right)$ and $T\left(w_{2}\right)$, respectively. To the empty word we associate the empty tree. Thus $T(w)$ is an increasing rooted planar binary tree, i.e. the distinction between left and right children is being made. For example, T(423516) is the tree shown in Figure 1.

To get the word $w$ back from the tree $T(w)$, simply read the labels of the nodes of $T(w)$ in topological order.

Next, we formulate some of the permutation properties from the above discussion in terms of the min-tree representation.

Lemma 7.3. A permutation $\sigma$ has no consecutive descents if and only if the tree $T(\sigma)$ has no node whose only child is a left child, except maybe for the rightmost node in topological order.

Proof. Write $\sigma=s_{1} s_{2} \cdots s_{n}$ and $T=T(\sigma)$. For convenience, we refer to the nodes of $T$ by their labels. We have $s_{i}>s_{i+1}$ if and only if $s_{i+1}$ is an ancestor of $s_{i}$ in $T$. Since $s_{i}$ and $s_{i+1}$ are consecutive nodes in the topological reading of $T$, it follows that $s_{i+1}$ is an ancestor of $s_{i}$ if and only if $s_{i}$ has no right child. Thus we have $s_{i}>s_{i+1}>s_{i+2}$ if and only if $s_{i+1}$ has no right child and $s_{i}$ is a descendant of $s_{i+1}$, i.e. $s_{i+1}$ has a lone left child. The proposition follows.

Proposition 7.4. A permutation $\sigma$ is in $\mathcal{R}_{n}$ if and only if the tree $T(\sigma)$ has no node whose only child is a left child.

Proof. We have $s_{n-1}>s_{n}$ if and only if the rightmost node $s_{n}$ has a (lone) left child. The proposition now follows from Lemma 7.3.

Proposition 7.5. A permutation $\sigma$ is in $\mathrm{SS}_{n}$ if and only if the rightmost node of $T(\sigma)$ is labeled n, no node has a lone left child, and for every node s not on the rightmost path (the path from the root to the rightmost node) that has both a left child $t$ and a right child $u$, the inequality $t>u$ holds. 


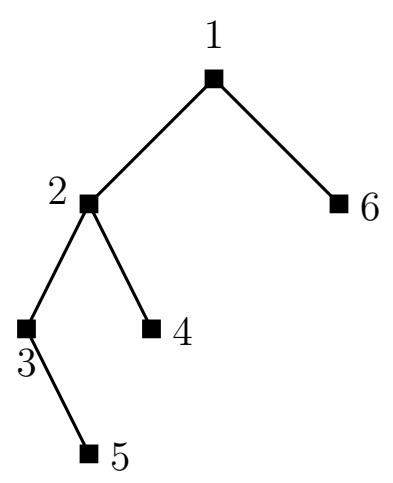

Figure 2: The tree $F_{2}(T(423516))$

Proof. If $T(\sigma)$ has a node $s$ not on the rightmost path whose left child $t$ is smaller than its right child $u$, then removing the elements of $\sigma$ that are greater than or equal to $u$ results in a permutation $\sigma^{\prime}$ such that in $T\left(\sigma^{\prime}\right)$, the node $s$ has a lone left child $t$ and is not the rightmost node, meaning that $\sigma^{\prime}$ contains a pair of consecutve descents, by Lemma 7.3. If on the other hand $T(\sigma)$ has no such node $s$, the removing $k$ largest elements of $\sigma$ does not create any nodes with a lone left child except maybe for the rightmost node.

One can see that for $\sigma=423516$, the tree $T(\sigma)$ shown in Figure 1 satisfies all conditions of Proposition 7.5, and hence $423516 \in \mathrm{SS}_{6}$. Next, we consider the sum of coefficients of $\hat{\Phi}_{n}(\mathbf{c},-\mathbf{d})$.

Theorem 7.6. The sum of coefficients of $\hat{\Phi}_{n}(\mathbf{c},-\mathbf{d})$ is $\left|\mathcal{R}_{n}\right|$.

Proof. The sum of coefficients of $\hat{\Phi}_{n}(\mathbf{c},-\mathbf{d})$ is $\hat{\Phi}_{n}(1,-1)=\Phi_{n}(1,2)$, which equals

$$
\sum_{\sigma \in \mathrm{SS}_{n}} 2^{d(\sigma)}
$$

where $d(\sigma)$ is the number of d's in $\operatorname{cd}(\sigma)$, or, equivalently, the number of descents of $\sigma$. Since the descents of $\sigma$ correspond to nodes of $T(\sigma)$ that have no right child (except for the rightmost node, which corresponds to the last element of $\sigma$ ), it follows from Proposition 7.4 that the descents of a permutation $\sigma \in \mathcal{R}_{n}$ correspond to the leaves of $T(\sigma)$ minus the rightmost node. Thus for $\sigma \in \mathcal{R}_{n}$ we have that $2^{d(\sigma)}$ is the number of leaves in $T(\sigma)$ minus one, which equals the number of of nodes of $T(\sigma)$ with two children. (The latter can be proved easily by induction.)

For a min-tree $T$ and a node $s$ of $T$ with two children, let $F_{s}(T)$ be the tree obtained by switching the left and the right subtrees of $T$. (This operation is called the Foata-Strehl action on the permutation encoded by $T$; see [4].) For example, if $T$ is the tree $T(423516)$ shown above, then $F_{2}(T)$ is the tree shown in Figure 2.

Note that the action of $F_{s}$ preserves the set of nodes with two children and does not create any nodes with a lone left child if the original tree contained no such nodes. 
Hence the set $T\left(\mathcal{R}_{n}\right)$ is invariant under this action. Observe also that the operators $F_{s}$ commute and satisfy $F_{s}^{2}=1$. Thus these operators, viewed as operators on permutations corresponding to trees, split the set $\mathcal{R}_{n}$ into orbits of size $2^{d(\sigma)}$, where $\sigma$ is any member of the orbit. It remains to show that each orbit contains exactly one permutation in $\mathrm{SS}_{n}$.

Given $\sigma \in \mathcal{R}_{n}$, there is a unique, up to order, sequence of operators $F_{s}$, where $s$ is on the rightmost path, that, when applied to $T(\sigma)$, makes $n$ the rightmost node of the resulting tree. An example is shown in Figure 3. (One needs to find the closest ancestor
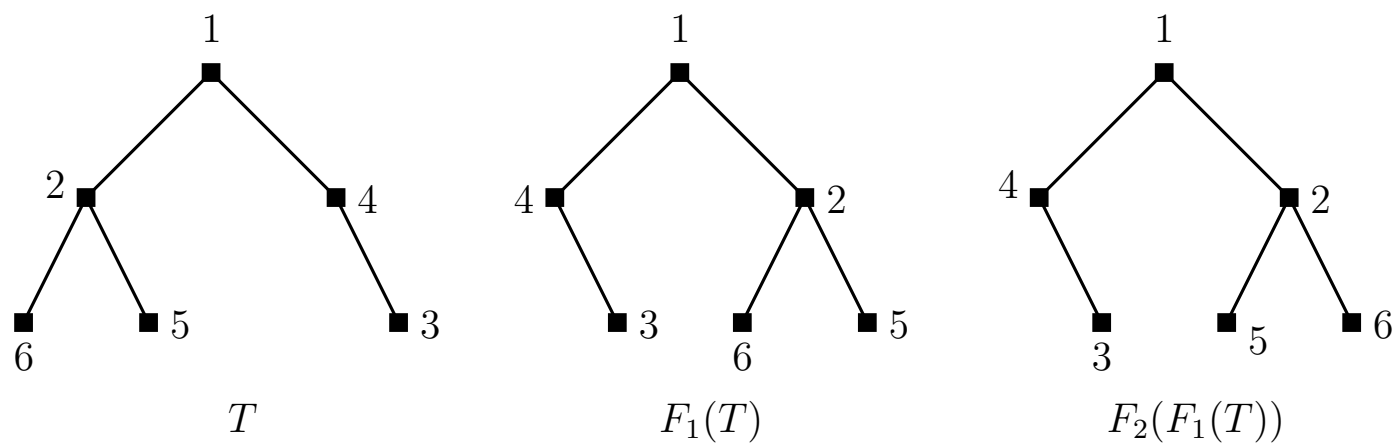

Figure 3: The action of $F_{1}$ and $F_{2}$ on a min-tree

of $n$ on the rightmost path and then apply the corresponding operator to bring the node $n$ closer to the rightmost path.) Once $n$ is the rightmost node, apply the operator $F_{s}$ to all nodes $s$ with two children for which the condition of Proposition 7.5 is violated. We obtain a tree corresponding to a permutation in $\mathrm{SS}_{n}$ in the orbit of $\sigma$. To see that each orbit contains only one member of $\mathrm{SS}_{n}$, observe that the action of $F_{s}$ preserves the sequence of elements on the path from 1 to $k$ for each $k$, and given the sequence of ancestors for each $k \in[n]$, there is a unique way of arranging the elements of $[n]$ to form a min-tree satisfying the conditions of Proposition 7.5: first, set the path from 1 to $n$ to be the rightmost path, and then set all lone children to be right children, and for all nodes with two children, set the greater element to be the left child.

The proof is now complete.

Table 2 lists the polynomials $\hat{\Phi}_{n}(\mathbf{c}, \mathbf{d})$ for $n \leq 6$.

\section{Shapiro-Woan-Getu permutations}

In this section we take a closer look at the class of permutations which we denoted by $\mathcal{R}_{n}$ in Section 7 . Recall that $\mathcal{R}_{n}$ is the set of permutations with no consecutive (double) descents and no descent at the end. They appear in the paper [8] by Shapiro, Woan, and Getu, hence the section title, who call them reduced permutations. The paper studies enumeration of permutations by the number of runs or slides, and in [7, Sec. 11.1] 


\begin{tabular}{l|l}
$n$ & $\hat{\Phi}_{n}(\mathbf{c}, \mathbf{d})$ \\
\hline 1 & 1 \\
2 & $\mathbf{c}$ \\
3 & $2 \mathbf{c}^{2}-\mathbf{d}$ \\
4 & $5 \mathbf{c}^{3}-2(\mathbf{c} \mathbf{d}+\mathbf{d} \mathbf{c})$ \\
5 & $16 \mathbf{c}^{4}-7\left(\mathbf{c}^{2} \mathbf{d}+\mathbf{d} \mathbf{c}^{2}\right)-5 \mathbf{c d} \mathbf{c}+4 \mathbf{d}^{2}$ \\
6 & $61 \mathbf{c}^{5}-26\left(\mathbf{c}^{3} \mathbf{d}+\mathbf{d} \mathbf{c}^{3}\right)-21\left(\mathbf{c} \mathbf{d} \mathbf{c}^{2}+\mathbf{c}^{2} \mathbf{d} \mathbf{c}\right)+10 \mathbf{d} \mathbf{c d}+12\left(\mathbf{c} \mathbf{d}^{2}+\mathbf{d}^{2} \mathbf{c}\right)$
\end{tabular}

Table 2: The polynomials $\hat{\Phi}_{n}(\mathbf{c}, \mathbf{d})$

Postnikov, Reiner, and Williams put these results in the context of structural properties of permutohedra: for instance, the polynomial encoding the distribution of permutations in $\mathcal{R}_{n}$ by the number of descents is the $\gamma$-polynomial of the classical permutohedron.

In Section 7 , we found the number $R_{n}$ of SWG permutations of size $n$ to be the sum of absolute values of coefficients of a $(\mathbf{c}, \mathbf{d})$-polynomial that, when expanded in terms of $\mathbf{a}$ and $\mathbf{b}$, gave the generating function for the alternating descent set statistic. Shapiro, Woan, and Getu provide a generating function for $R_{n}$ :

$$
R(x):=\sum_{n \geq 0} R_{n} \cdot \frac{x^{n}}{n !}=1+\frac{2 \tan (x \sqrt{3} / 2)}{\sqrt{3}-\tan (x \sqrt{3} / 2)}
$$

(we put $R_{0}=1$ ). Observe that $R(x) R(-x)=1$, a property that $R(x)$ shares with $e^{x}$ and $\tan x+\sec x$, which are the two fundamental generating functions in the analisys done in previous sections. There is a further resemblance with the Euler numbers $E_{n}$ if one looks at the logarithm of $R(x)$ :

$$
\ln (R(x))=-x+2 \sum_{n \geq 0} R_{2 n} \cdot \frac{x^{2 n+1}}{(2 n+1) !}
$$

Comparing with

$$
\ln (\tan x+\sec x)=\sum_{n \geq 0} E_{2 n} \cdot \frac{x^{2 n+1}}{(2 n+1) !},
$$

we see that taking the logarithm has a similar effect on both $R(x)$ and $\tan x+\sec x$ of taking the even part and integrating, except that for $R(x)$ all coefficients excluding that of $x$ are doubled.

The fact that

$$
\int \sec x d x=\ln (\tan x+\sec x)
$$

(omitting the arbitrary constant of integration) has been used in the proof of Theorem 4.3. This textbook integral formula can be proved combinatorially using the exponential formula for generating functions (see [13, Sec. 5.1]). Given an up-down permutation $\sigma$, divide 
$\sigma$ into blocks by the following procedure. Put the subword of $\sigma$ starting at the beginning of $\sigma$ and ending at the element equal to 1 in the first block, and remove this block from $\sigma$. In the resulting word, find the maximum element $m_{2}$ and put the subword consisting of initial elements of the word up to, and including, $m_{2}$ in the second block, and remove the second block. In the remaining word, find the minimum element $m_{3}$, and repeat until there is nothing left, alternating between cutting at the minimum and at the maximum element of the current word. For example, for $\sigma=593418672$, the blocks would be 59341, 8 , and 672 . Note that given the blocks one can uniquely recover the order in which they must be concatenated to form the original permutation $\sigma$. Indeed, the first block is the one containing 1 , the second block contains the largest element not in the the first block, the third block contains the smallest element not in the first two blocks, and so on. Thus to construct an up-down permutation of size $n$ we need to divide the elements of $[n]$ into blocks of odd size, then determine the order of concatenation using the above principle, and then arrange the elements of odd numbered blocks in up-down order and those of even numbered blocks in down-up order. There are $E_{k-1}$ ways to arrange the elements in a block of size $k$ for odd $k$, and 0 ways for even $k$ since we do not allow blocks of even size. Thus (27), which is equivalent to (28), follows from the exponential formula. This argument "combinatorializes" the proof of Theorem 4.3. It would be nice to give a similar argument for reduced permutations $\mathcal{R}_{n}$.

Problem 8.1. Find a combinatorial proof of formula (26) for $\ln (R(x))$.

Another problem emerging from the results of Section 7 is the following.

Problem 8.2. Give a combinatorial interpretation of the coefficients of the polynomial $\hat{\Phi}_{n}(\mathbf{c},-\mathbf{d})$ by partitioning the set $\mathcal{R}_{n}$ into classes corresponding to the $F_{n-1}$ monomials.

It is worth pointing out here that even though one can split $\mathcal{R}_{n}$ into $F_{n-1}$ classes corresponding to $(\mathbf{c}, \mathbf{d})$-monomials by descent set, like it was done for simsun permutations in Section 7, the resulting polynomial is different from $\hat{\Phi}_{n}(\mathbf{c},-\mathbf{d})$. There are a few hints on what the correct way to refine permutations in $\mathcal{R}_{n}$ could be. The coefficient of $\mathbf{c}^{n-1}$ in $\hat{\Phi}_{n}(\mathbf{c},-\mathbf{d})$ is the Euler number $E_{n}$, and the set $\mathcal{R}_{n}$ includes at least three kinds of permutations mentioned in this paper that are counted by $E_{n}$ : alternating permutations ending with an ascent, simsun permutations, and permutations $\sigma \in \mathfrak{S}_{n}$ such that $\sigma \circ(n+1)$ has no odd 3 -factors. Values of $\hat{\Phi}_{n}$ for small $n$, including those listed in Table 2, present evidence that the common coefficient of $\mathbf{c}^{n-3} \mathbf{d}$ and $\mathbf{d} \mathbf{c}^{n-3}$ is $\hat{A}(n-1,2)$ (the number of permutations of size $n-1$ with exactly one alternating descent).

\section{A q-analog of Euler numbers}

Let $\hat{A}_{n}(t, q)$ denote the bivariate polynomial of Theorem 3.7:

$$
\hat{A}_{n}(t, q):=\sum_{\sigma \in \mathfrak{S}_{n}} t^{\hat{d}(\sigma)} q^{\hat{\imath}(\sigma)} .
$$


Then the alternating Eulerian polynomial $\hat{A}_{n}(t)$ is just the specialization $t \hat{A}_{n}(t, 1)$. We also noted earlier (Corollary 3.5) that

$$
\hat{A}_{n}(1, q)=[n]_{q} !
$$

the classical $q$-analog of the factorial defined by $[n]_{q} !:=[1]_{q}[2]_{q} \cdots[n]_{q}$, where $[i]_{q}:=$ $1+q+q^{2}+\cdots+q^{i-1}$. One can ask about other specializations of $\hat{A}_{n}(t, q)$, such as the ones with $t$ or $q$ set to 0 . Clearly, we have $\hat{A}_{n}(t, 0)=1$ because the only permutation $\sigma \in \mathfrak{S}_{n}$ for which $\hat{\imath}(\sigma)=0$ also satisfies $\hat{d}(\sigma)=0$. The case of $t=0$ is more curious and is the subject of this section.

We have $\hat{d}(\sigma)=0$ if and only if $\sigma$ is an up-down permutation. Thus $\hat{A}_{n}(0,1)=E_{n}$, and the specialization $\hat{A}_{n}(0, q)$ gives a $q$-analog of the Euler number $E_{n}$ with coefficients encoding the distribution of the number of alternating inversions among up-down permutations. The following lemma is key in understanding this $q$-analog.

Lemma 9.1. For a permutation $\sigma \in \mathfrak{S}_{n}$, let $\operatorname{code}(\sigma)=\left(\hat{c}_{1}, \hat{c}_{2}, \ldots, \hat{c}_{n-1}\right)$. Then $\sigma$ is up-down (resp., down-up) if and only if $\hat{c}_{i}+\hat{c}_{i+1} \leq n-1-i$ (resp., $\hat{c}_{i}+\hat{c}_{i+1} \geq n-i$ ) for all $i$.

Proof. This fact is just a special case of Lemma 3.9.

For various reasons it is more convenient to study the distribution of $\hat{\imath}$ on downup, rather than up-down, permutations. The $q$-analog obtained this way from down-up permutations is essentially equivalent to $\hat{A}_{n}(0, q)$, the difference being the reverse order of coefficients and a power of $q$ factor. It follows from Lemma 9.1 that for a down-up permutation $\sigma \in \mathfrak{S}_{n}$, we have

$$
\hat{\imath}(\sigma) \geq(n-1)+(n-3)+(n-5)+\cdots=\left\lfloor\frac{n^{2}}{4}\right\rfloor .
$$

Therefore let $\mathrm{Alt}_{n}$ be the set of down-up permutations in $\mathfrak{S}_{n}$, and define

$$
\hat{E}_{n}(q):=q^{-\left\lfloor n^{2} / 4\right\rfloor} \sum_{\sigma \in \operatorname{Alt}_{n}} q^{\hat{\imath}(\sigma)} .
$$

The values of $\hat{E}_{n}(q)$ for small $n$ are given in Table 3. We have the following facts about $\hat{E}_{n}(q)$.

Proposition 9.2. (a) The polynomial $\hat{E}_{n}(q)$ is monic and has degree $\left\lfloor\frac{(n-1)^{2}}{4}\right\rfloor$.

(b) $\hat{A}_{n}(0, q)=q^{\left\lfloor(n-1)^{2} / 4\right\rfloor} \cdot \hat{E}_{n}\left(q^{-1}\right)$.

(c) $\hat{E}_{n}(0)=c_{\lfloor n / 2\rfloor}$, the $\lfloor n / 2\rfloor$-th Catalan number. 


\begin{tabular}{l|l}
$n$ & $\hat{E}_{n}(q)$ \\
\hline $0,1,2$ & 1 \\
3 & $1+q$ \\
4 & $2+2 q+q^{2}$ \\
5 & $2+5 q+5 q^{2}+3 q^{3}+q^{4}$ \\
6 & $5+12 q+16 q^{2}+14 q^{3}+9 q^{4}+4 q^{5}+q^{6}$ \\
7 & $5+21 q+42 q^{2}+56 q^{3}+56 q^{4}+44 q^{5}+28 q^{6}+14 q^{7}+5 q^{8}+q^{9}$
\end{tabular}

Table 3: The polynomials $\hat{E}_{n}(q)$ for $n \leq 7$

Proof. (a) By Proposition 3.4, the unique permutation $\sigma \in \mathfrak{S}_{n}$ with the maximum possible number of alternating inversions is the one for which code $(\sigma)=(n-1, n-2, \ldots, 1)$. By Lemma 9.1, or by simply realizing that $\sigma=n \circ 1 \circ(n-1) \circ 2 \circ \cdots$, one can see that $\sigma \in \mathrm{Alt}_{n}$. We have $\hat{\imath}(\sigma)=n(n-1) / 2$, and thus the degree of $E_{n}(q)$ is $n(n-1) / 2-\left\lfloor n^{2} / 4\right\rfloor=$ $\left\lfloor(n-1)^{2} / 4\right\rfloor$.

(b) This identity is an algebraic restatement of an earlier observation.

(c) The constant term $\hat{E}_{n}(0)$ of $\hat{E}_{n}(q)$ is the number of permutations $\sigma \in \mathrm{Alt}_{n}$ with exactly $\left\lfloor n^{2} / 4\right\rfloor$ alternating inversions. By (29), these are precisely the permutations in Alt $_{n}$ satisfying $\hat{c}_{i}+\hat{c}_{i+1}=n-i$ for odd $i$. Let $\sigma \in \mathrm{Alt}_{n}$ be a permutation with this property.

For $j \geq 1$, we have $\hat{c}_{2 j} \geq n-2 j-\hat{c}_{2 j+1}=\hat{c}_{2 j+2}-1$. Thus $\hat{c}_{2}, \hat{c}_{4}, \ldots, \hat{c}_{2\lfloor n / 2\rfloor}$ is a strictly decreasing sequence of non-negative integers satisfying $\hat{c}_{2 j} \leq n-2 j$ (for convenience, let $\left.\hat{c}_{n}=0\right)$. Reversing the sequence and reducing the $k$-th term by $k-1$ for all $k$ yields a bijective correspondence with sequences of $\lfloor n / 2\rfloor$ non-negative integers whose $k$-th term does not exceed $k-1$, and it is well known that there are $c_{\lfloor n / 2\rfloor}$ such sequences. Since $\hat{c}_{2 j-1}$ is uniquely determined by $\hat{c}_{2 j}$, it follows that there are $c_{\lfloor n / 2\rfloor}$ permutations $\sigma \in \mathrm{Alt}_{n}$ with $\left\lfloor n^{2} / 4\right\rfloor$ alternating inversions.

It is curious to note that the permutations in Alt $_{n}$ with $\left\lfloor n^{2} / 4\right\rfloor$ alternating inversions can be characterized in terms of pattern avoidance, so that Proposition 9.2(c) follows from a result of Mansour [6] stating that the number of 312-avoiding down-up permutations of size $n$ is $c_{\lfloor n / 2\rfloor}$.

Proposition 9.3. A permutation $\sigma \in \operatorname{Alt}_{n}$ has $\hat{\imath}(\sigma)=\left\lfloor n^{2} / 4\right\rfloor$ if and only if $\sigma$ is 312avoiding.

The following lemma implies the above proposition and is useful in the later discussion as well.

Lemma 9.4. For a permutation $\sigma=\sigma_{1} \sigma_{2} \cdots \sigma_{n} \in \operatorname{Alt}_{n}$, the number $\hat{\imath}(\sigma)$ is equal to 
$\left\lfloor n^{2} / 4\right\rfloor$ plus the number of occurrences of the generalized pattern 31-2 (that is, the number of pairs of indices $i<j$ such that $\sigma_{i+1}<\sigma_{j}<\sigma_{i}$ ).

Proof. For $i \in[n-1]$, define

$$
S_{i}:= \begin{cases}\left\{j \mid j>i \text { and } \sigma_{i}>\sigma_{j}\right\} & \text { if } i \text { is odd } \\ \left\{j \mid j>i \text { and } \sigma_{i}<\sigma_{j}\right\} & \text { if } i \text { is even }\end{cases}
$$

Thus $\hat{c}_{i}=\left|S_{i}\right|$. Let $i$ be odd. Then $\sigma_{i}>\sigma_{i+1}$, so $i+1 \in S_{i}$ and for every $j>i+1$, either $\sigma_{j}<\sigma_{i}$ or $\sigma_{j}>\sigma_{i+1}$, or both. Hence $\{i+1, i+2, \ldots, n-1\} \subseteq S_{i} \cup S_{i+1}$ and $\hat{c}_{i}+\hat{c}_{i+1}=n-1-i+\left|S_{i} \cap S_{i+1}\right|$. But $S_{i} \cap S_{i+1}$ is the set of indices $j>i+1$ such that $\sigma_{i+1}<\sigma_{j}<\sigma_{i}$, i.e. the number of occurrences of the pattern 31-2 beginning at position $i$. Therefore the total number of alternating inversions is $\sum_{i \text { odd }}(n-1-i)=\left\lfloor n^{2} / 4\right\rfloor$ plus the total number of occurrences of 31-2.

Proof of Proposition 9.3. Suppose that a permutation $\sigma \in \operatorname{Alt}_{n}$ has exactly $\left\lfloor n^{2} / 4\right\rfloor$ alternating inversions but is not 312-avoiding. Choose a triple $i<k<j$ such that $\sigma_{k}<\sigma_{j}<\sigma_{i}$ and the difference $k-i$ is as small as possible. Suppose that $k-i \geq 2$. If $\sigma_{k-1}<\sigma_{j}$, then we have $\sigma_{k-1}<\sigma_{j}<\sigma_{i}$, contradicting the choice of $i, k$, and $j$. If $\sigma_{k-1}>\sigma_{j}$, then we have $\sigma_{k}<\sigma_{j}<\sigma_{k-1}$, also contradicting the choice of $i, k$, and $j$. Hence $k=i+1$, and we obtain a contradiction by Lemma 9.4.

In view of Lemma 9.4 , we can write $\hat{E}_{n}(q)$ as

$$
\hat{E}_{n}(q)=\sum_{\sigma \in \mathrm{Alt}_{n}} q^{31-2(\sigma)}
$$

where $31-2(\sigma)$ is the number of occurrences of 31-2 in $\sigma$. In what follows, we use this expression to show how a $q$-analog of a combinatorial identity representing the Euler number $E_{n}$ as a weighted sum of Dyck paths yields a refined identity of $\hat{E}_{n}(q)$.

First, we need to introduce Dyck paths, which are perhaps the most famous combinatorial objects counted by Catalan numbers. A Dyck path of length $2 m$ is a continuous path consisting of line segments, or steps, each of which connects an integer point $(x, y)$ with either $(x+1, y-1)$ or $(x+1, y+1)$, such that the path starts at $(0,0)$, ends at $(2 m, 0)$, and never goes below the $x$-axis, that is, contains no point with a negative $y$-coordinate. The identity we are about to describe involves associating a certain weight with every step of a Dyck path, defining the weight of the entire path to be the product of the weights of the individual steps, and adding the weights of all Dyck paths of length $2 m$ to obtain $E_{2 m}$ or $E_{2 m+1}$, or, in the case of the refined identity, $\hat{E}_{2 m}(q)$ or $\hat{E}_{2 m+1}(q)$.

For a step in a Dyck path, define the level of that step to be the $y$-coordinate of the highest point of the corresponding segment of the path. Given a Dyck path $\mathcal{D}$ of length $2 m$, let $\ell(i)$ be the level of the $i$-th step of $\mathcal{D}$. Define

$$
w_{\mathcal{D}, i}^{e}(q):=[\ell(i)]_{q}
$$




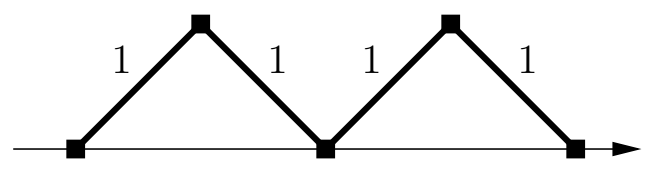

$w^{e}=1$

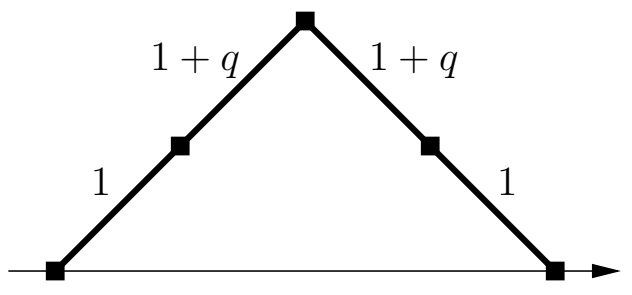

$w^{e}=(1+q)^{2}$

Figure 4: Weighted Dyck paths adding up to $\hat{E}_{4}(q)$

and

$$
w_{\mathcal{D}, i}^{o}(q):= \begin{cases}{[\ell(i)]_{q},} & \text { if the } i \text {-th step is an up-step; } \\ {[\ell(i)+1]_{q},} & \text { if the } i \text {-th step is a down-step }\end{cases}
$$

(the letters $e$ and $o$ stand for even and odd, as will become clear shortly). As mentioned above, we set the weight of the entire path to be the product of step weights:

$$
\begin{aligned}
& w_{\mathcal{D}}^{e}(q)=\prod_{i=1}^{2 m} w_{\mathcal{D}, i}^{e}(q) ; \\
& w_{\mathcal{D}}^{o}(q)=\prod_{i=1}^{2 m} w_{\mathcal{D}, i}^{o}(q) .
\end{aligned}
$$

Theorem 9.5. We have

$$
\sum_{\mathcal{D}} w_{\mathcal{D}}^{e}(q)=\hat{E}_{2 m}(q)
$$

and

$$
\sum_{\mathcal{D}} w_{\mathcal{D}}^{o}(q)=\hat{E}_{2 m+1}(q)
$$

where both sums are taken over all Dyck paths of length $2 m$.

For example, for $m=2$ there are two Dyck paths, shown in Figures 4 and 5 with step weights given by $w_{\mathcal{D}, i}^{e}(q)$ and $w_{\mathcal{D}, i}^{o}(q)$. From these weighted paths, we get

$$
1+(1+q)^{2}=2+2 q+q^{2}=\hat{E}_{4}(q)
$$

and

$$
(1+q)^{2}+(1+q)^{2}\left(1+q+q^{2}\right)=2+5 q+5 q^{2}+3 q^{3}+q^{4}=\hat{E}_{5}(q) .
$$

In the classical case $q=1$, the identities of Theorem 9.5 are due to Françon and Viennot [2], and are discussed in a broader context in the book [3, Sec. 5.2] by Goulden and Jackson. The proof of our identities is a refinement of the original argument. 


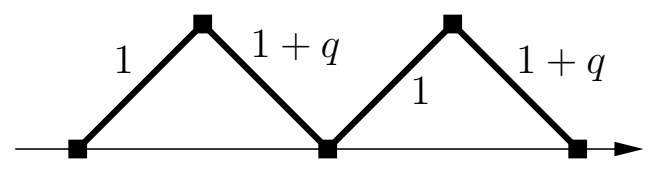

$w^{o}=(1+q)^{2}$

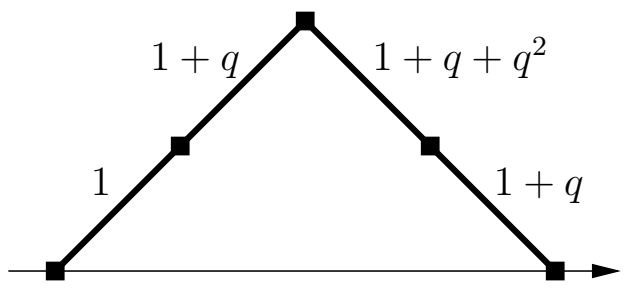

$w^{o}=(1+q)^{2}\left(1+q+q^{2}\right)$

Figure 5: Weighted Dyck paths adding up to $\hat{E}_{5}(q)$

Proof of Theorem 9.5. Fix a positive integer $n>1$, and let $m=\lfloor n / 2\rfloor$. Recall that in Section 7 we associated to a permutation $\sigma \in \mathfrak{S}_{n}$ an increasing planar binary tree $T(\sigma)$ with vertex set $[n]$. Extending the argument in the proof of Lemma 7.3, we conclude that $\sigma$ is in $\mathrm{Alt}_{n}$ if and only if the tree $T(\sigma)$ has no vertices with a lone child, except for the rightmost vertex in the case of even $n$, which has a lone left child. For $\sigma \in \mathrm{Alt}_{n}$, define the corresponding Dyck path $\mathcal{D}(\sigma)$ of length $2 m$ as follows: set the $i$-th step of the path to be an up-step if vertex $i$ of $T(\sigma)$ has at least one child, and set the $i$-th step to be a down-step if vertex $i$ is a leaf of $T(\sigma)$. We leave it as an exercise for the reader to check that $\mathcal{D}(\sigma)$ is a valid Dyck path.

Fix a Dyck path $\mathcal{D}$ of length $2 m$. We claim that

$$
\sum_{\sigma \in \operatorname{Alt}_{n}: \mathcal{D}(\sigma)=\mathcal{D}} q^{31-2(\sigma)}= \begin{cases}w_{\mathcal{D}}^{e}(q), & \text { if } n \text { is even; } \\ w_{\mathcal{D}}^{o}(q), & \text { if } n \text { is odd. }\end{cases}
$$

To prove the claim, consider for every $i$ the subtree $T_{i}(\sigma)$ obtained from $T(\sigma)$ by removing all vertices labeled with numbers greater than $i$. For the sake of clarity, one should imagine the "incomplete" tree $T_{i}(\sigma)$ together with "loose" edges indicating those edges with parent vertices in $T_{i}(\sigma)$ that appear when $T_{i}(\sigma)$ is completed to $T(\sigma)$. For even $n$ one should also think of a loose edge directed to the right coming out of the rightmost vertex of every tree $T_{i}(\sigma)$ including $T_{n}(\sigma)=T(\sigma)$ - this way the number of edges coming out of a vertex of $T_{i}(\sigma)$ is always 0 or 2 .

Observe that for $1 \leq i \leq 2 m$, the number of loose edges of $T_{i}(\sigma)$ is equal to $y_{\mathcal{D}}(i)+1$, where $y_{\mathcal{D}}(i)$ is the $y$-coordinate of the point of $\mathcal{D}$ whose $x$-coordinate is $i$. Indeed, $T_{1}(\sigma)$ has two loose edges, and $T_{i+1}(\sigma)$ is obtained from $T_{i}(\sigma)$ by attaching a non-leaf to a loose edge, thus increasing the number of loose edges by one, if the $i$-th step of $\mathcal{D}$ is an up-step, or by attaching a leaf to a loose edge, thus reducing the number of loose edges by one, if the $i$-th step is a down-step. Hence we can count the number of permutations $\sigma \in \mathrm{Alt}_{n}$ with $\mathcal{D}(\sigma)=\mathcal{D}$ by multiplying together the number of possibilities to attach a vertex labeled $i+1$ to $T_{i}(\sigma)$ to form $T_{i+1}(\sigma)$ for all $1 \leq i \leq n-1$. The number of valid places to attach vertex $i+1$ is equal to the number of loose edges in $T_{i}(\sigma)$ unless $i+1$ is a leaf of $T(\sigma)$ and $n$ is even, in which case we have one fewer possibilities, because we are not 

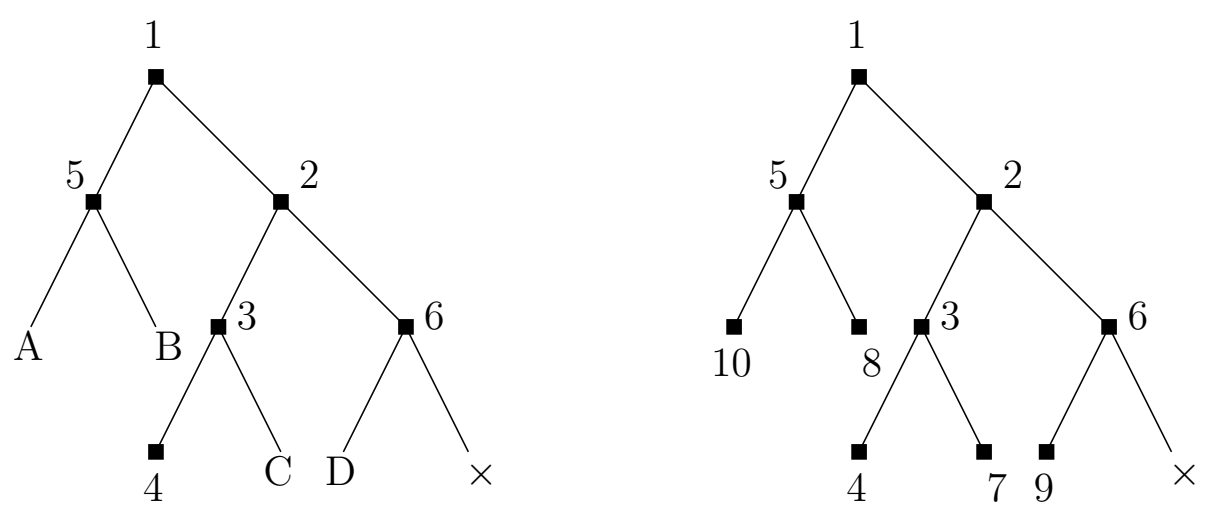

Figure 6: An intermediate tree $T_{6}(\sigma)$ and its completion $T(\sigma)$

allowed to make the rightmost vertex a leaf. Note that the level $\ell(i)$ of the $i$-th step of $\mathcal{D}$ is equal to $y_{\mathcal{D}}(i)$ if it is an up-step, or $y_{\mathcal{D}}(i)+1$ if it is a down-step. Comparing with the choice of step weights, we conclude that the number of possibilities to attach vertex $i+1$ is $w_{\mathcal{D}, i+1}^{e}(1)$ if $n$ is even, or $w_{\mathcal{D}, i+1}^{o}(1)$ if $n$ is odd. (For odd $n$ and $i=n-1$ the latter assertion makes no sense as $\mathcal{D}$ does not have an $n$-th step; however, there is just one way to attach the last vertex, so the counting argument is not affected.)

The above computation proves the $q=1$ case of (30). To prove the general claim, we need to show that if there are $p$ possibilities to attach vertex $i+1$ to a loose edge of $T_{i}(\sigma)$, then the number of occurrences of the 31-2 pattern "induced" by the attachment is 0 for one of the possibilities, 1 for another possibility, 2 for another, and so on, up to $p-1$. Then choosing a place to attach vertex $i+1$ would correspond to choosing a term from $1+q+q^{2}+\cdots+q^{p-1}=[p]_{q}$, the weight of the $i$-th step of $\mathcal{D}$, which is a factor in the total weight of $\mathcal{D}$, and (30) would follow.

It remains to specify which occurrences of 31-2 in $\sigma$ are induced by which vertex of $T(\sigma)$. Suppose there are $p$ possible places to attach vertex $i+1$. Order these places according to the topological order of tree traversal, and suppose we choose to put vertex $i+1$ in the $k$-th place in this order. Let $r_{1}, r_{2}, \ldots, r_{k-1}$ be the numbers of the vertices immediately following the first $k-1$ places in the topological order, and let $a_{j}$ denote the label of the rightmost vertex of the eventual subtree of $T(\sigma)$ rooted at what is currently the $j$-th of these $k-1$ places. Although $a_{j}$ is not determined at the time vertex $i+1$ is attached, it is certain that $r_{j}<i+1<a_{j}$ and that $a_{j}$ and $r_{j}$ will be consecutive elements of $\sigma$, with $i+1$ located somewhere to the right, resulting in an occurrence of 31-2. Thus the choice to put vertex $i+1$ in the $k$-th available place induces $k-1$ occurrences of $31-2$, one for each $1 \leq j \leq k-1$. It is not hard to check that each occurrence of 31-2 is induced by some vertex of $T(\sigma)$, namely, the vertex corresponding to the rightmost element forming the pattern, in the way described above.

Let us illustrate the argument with an example. The left side of Figure 6 shows the tree $T_{6}(\sigma)$ for some $\sigma \in \mathrm{Alt}_{10}$, with the four potential places for vertex 7 marked $\mathrm{A}, \mathrm{B}$, 
$\mathrm{C}$, and D. If vertex 7 is put in position $\mathrm{A}$, then it induces no occurrences of $31-2$. If it is put in position $\mathrm{B}$, it induces one occurrence of 31-2 as the triple $a 5-7$ is created, where $a$ stands for the number of the rightmost vertex in the subtree rooted at $\mathrm{A}$ in the eventual tree. If vertex 7 is put in position $\mathrm{C}$, then in addition to the triple $a 5-7$, one obtains a second 31-2 triple b1-7. Finally, putting vertex 7 in position D results in a third 31-2 triple $c 2-7$. (Here $b$ and $c$ are defined by analogy with $a$.) On the right side of Figure 6 we have a possible completion of the tree on the left, which corresponds to the permutation $\sigma=10581437296$.

The theorem now follows by taking the sum of (30) over all Dyck paths $\mathcal{D}$ of length $2 m$.

\section{Acknowledgments}

This paper is part of the author's Ph.D. thesis, and the research presented here was carried out in the Department of Mathematics at the Massachusetts Institute of Technology. I would like to thank Pavlo Pylyavskyy for his ideas and conversations that led to this work. I am also grateful to Richard Stanley and Alex Postnikov for helpful discussions.

\section{References}

[1] Babson, E. and Steingrímsson, E.: Generalized Permutation Patterns and a Classification of the Mahonian Statistics, Séminaire Lotharingien de Combinatoire B44b (2000)

[2] Françon, J. and Viennot, G.: Permutations selon leurs pics, creux, doubles montées et double descentes, nombres d'Euler et nombres de Genocchi, Discrete Math. 28 (1979), no. 1, pp. 21-35

[3] Goulden, I.P. and Jackson, D.M.: Combinatorial Enumeration, John Wiley \& Sons (1983)

[4] Hetyei, G. and Reiner, E.: Permutation Trees and Variation Statistics, Europ. J. Combinatorics 19 (1998), pp. 847-866

[5] Kitaev, S. and Mansour, T.: Simultaneous Avoidance of Generalized Patterns, Ars. Combinatoria 75 (2005), pp. 267-288

[6] Mansour, T.: Restricted 132-alternating Permutations and Chebyshev Polynomials, Ann. Comb. 7 (2003), no. 2, pp. 201-227

[7] Postnikov, A., Reiner, V., and Williams, L.: Faces of Generalized Permutohedra, preprint, math.C0/0609184

[8] Shapiro, L., Woan, W.J., and Getu, S.: Runs, Sildes and Moments, SIAM J. Algebraic Discrete Mehtods 4 (1983), pp. 459-466

THE ELECTRONIC JOURNAL OF COMBINATORICS 15 (2008), \#R132 
[9] Shareshian, J. and Wachs, M.: q-Eulerian Polynomials: Excedance Number and the Major Index, Electron. Res. Announc. Amer. Math. Soc. 13 (2007), pp. 33-45

[10] Stanley, R.P.: Binomial Posets, Möbius Inversion, and Permutation Enumeration, $J$. Combinatorial Theory Ser. A 20 (1976), pp. 336-356

[11] Stanley, R.P.: A Survey of Eulerian Posets, Polytopes: Abstract, Convex, and Computational, NATO ASI Series C, vol. 440, Kluwer Academic Publishers, Dordrecht/Boston/London (1994), pp. 301-333

[12] Stanley, R.P.: Enumerative Combinatorics, Vol. 1, Cambridge University Press (1997)

[13] Stanley, R.P.: Enumerative Combinatorics, Vol. 2, Cambridge University Press (1997) 